\title{
Nouvelles recherches archéologiques sur l'âge du Fer IIA à Tell el-Far'a, Palestine
}

\section{New archaeological research on Iron Age IIA at Tell el-Far'a, Palestine}

\author{
JUAN-LUIS MONTERO FENOLLÓS \\ Departamento de Humanidades \\ Universidade da Coruña \\ Campus de Esteiro, 15403 Ferrol (A Coruña) \\ fenollos@udc.es \\ https://orcid.org/0000-0002-0689-3765
}

\author{
FRANCISCO CARAMELO \\ Departamento de História / NOVA FCSH \\ Universidade NOVA de Lisboa \\ Avenida de Berna, 26 C, 1069-061 Lisboa \\ francisco.caramelo@fcsh.unl.pt \\ https://orcid.org/0000-0001-5865-1699
}

\begin{abstract}
Résumé
Après les travaux pionniers (1946-1960) du père Roland de Vaux, directeur de l'École biblique et archéologique française de Jérusalem, à Tell el-Far'a, au nord-est de la ville de Naplouse (Palestine), un nouveau projet conjoint international de recherche archéologique a été lancé en 2017 par l'Université de La Corogne, l'Université NOVA de Lisbonne et le Ministère du Tourisme et des Antiquités de Palestine. Les trois campagnes menées à ce jour ont fourni de nouvelles données sur l'âge du Fer IIA, en particulier sur la datation absolue d'une maison de la période VIIb du site. Pour le moment, il ne s'agit que de cinq dates par radiocarbone (AMS) obtenues pour cette période. Ce sont les premières que nous avons de ce site, clé pour avancer sur la question complexe de la chronologie absolue en Palestine au début du ler millénaire av. J.-C. Bien que les données ne doivent pas être considérées comme définitives, elles indiquent une date du $\mathrm{X}^{\mathrm{C}}$ siècle av. J.-C. pour la « Maison A », qui a été fouillée en 2017-2019 à Tell el-Far'a (VIlb).
\end{abstract}

Mots clé: Palestine, Tell el-Far‘a, âge du Fer II, Tirza, datation absolue

\section{Abstract}

Through pioneering work (1946-1960) of Father Roland de Vaux, former director of the École biblique et archéologique française in Jerusalem at Tell el-Far'a, north east of Nablus (Palestine), the new international and joint archaeological research Project was launched in 2017, led by the University of A Coruña, the NOVA University, Lisbon, and the Ministry of Tourism and Antiquities of Palestine. Until the present day, the three archaeological seasons have revealed new data concerning Iron Age IIA, particularly the absolute dating related with a house of period VIIb of the site. Until now, we are dealing with only five dates of radiocarbon (AMS) concerning that same period, the first ones concerning Tell el-Far'a, key data to the complex issue of absolute chronologie in Palestine at the beginning of $1^{\text {st }}$ millennium BC. Not being definitive, these data, however, refer to a date belonging to the $10^{\text {th }}$ century BC related with "House A", excavated in 2017-2019 at Tell el-Far'a (VIIb). Key words: Palestine, Tell el-Far'a, Iron Age II, Tirzah, absolute date 


\section{Le contexte géographique}

Tell el-Far'a est situé à la tête du wadi el-Far'a, dans les montagnes centrales de la Palestine (Samarie dans la tradition biblique). Le site se trouve à Ir $\mathrm{km}$ au nord-est de la ville de Naplouse (Cisjordanie), mais il appartient aujourd'hui administrativement à la province de Tubas (figure I).

Le wadi el-Far'a, à $50 \mathrm{~km}$ environ à l'est de la mer Méditerranée, est le plus grand affluent du Jourdain et il est considéré comme l'un des wadis les plus importants de la Cisjordanie (Sharkas et alii 2005: 19). Il est alimenté principalement par les eaux de l'Aïn el-Far'a, que V. Guérin (I874: 234-235 et 260) a défini comme l'une des sources les plus belles et les plus importantes de Palestine :

Les bords de ce torrent, dont la source est abondante et intarissable, sont couverts de superbes touffes de lauriers-roses en fleur, de roseaux gigantesques et de verdoyants seder, qui forment un épais ombrage sous lequel coule un ruisseau éternellement murmurant.

D'un point de vue géologique, le wadi el-Far'a fait partie de la grande région du rift de la mer Morte et du système structurel complexe de l'Anticline d'el-Far'a. La lithologie primaire de la région est caractérisée par la présence de diverses formes de calcaire (Sharkar et alii, 2005: 27). Sa longueur, sa pente modérée, son sol fertile et son climat méditerranéen semi-aride font que les terres environnantes sont parmi les plus riches et les mieux irriguées de la région. L'irrigation, aujourd'hui comme dans le passé, repose sur un système de canaux et d'aqueducs.

Le wadi el-Far'a, qui a une longueur de $37 \mathrm{~km}$ entre 'Aïn el-Far'a et le Jourdain, près de Jisr Damaniya, était également une voie de communication importante dans l'antiquité, reliant Tell el-Far'a (peut-être l'ancienne Tirza) à la vallée du Jourdain. A. Zertal (2008: 26-28) a identifié cet itinéraire avec la « route ouest » citée dans Dt II, 3 O.

Tell el-Far'a est stratégiquement situé dans ce contexte géographique. C'est un tell de dimensions importantes situé sur un plateau rocheux de $198 \mathrm{~m}$ d'altitude. Il mesure $550 \mathrm{~m}$ de long sur $400 \mathrm{~m}$ de large, ce qui correspond à une superficie d'environ 22 hectares. Son implantation à cet endroit est due à trois raisons essentielles : une défense naturelle facile (sauf pour le secteur ouest) ; un bon approvisionnement en eau grâce à deux sources naturelles proches ('Aïn ed-Dulaib et 'Aïn el-Far'a); et un contrôle de la voie de communication reliant la vallée du Jourdain (située à $20 \mathrm{~km}$ à l'ouest) à la région de l'ancienne ville de Sichem (aujourd'hui Tell Balata, à Naplouse).

\section{Premières recherches à Tell el-Far'a et sa région}

Le XIX ${ }^{\mathrm{e}}$ siècle a été l'âge d'or des explorateurs qui ont voyagé en Terre Sainte, désireux de connaître la géographie biblique sur le terrain. Le premier d'entre eux fut le bibliste américain E. Robinson, qui, en 1838 , parcourut une grande partie de la Palestine. Dans l'ouvrage qu'il a publié plus tard avec les détails de son voyage, il fait référence au «Wady al-Fâri'a », un affluent de la rive occidentale du Jourdain qu'il place entre les villes de Tubas, à son avis à identifier avec la ville biblique de Thèbez, et Naplouse (Robinson, I84r: ror et 158).

V. Guérin a été le premier à mener à bien une exploration systématique de la géographie palestinienne. Dans son exposé concernant la région de Samarie, qu'il a visitée en I870, l'auteur français nous fournit la description qui est considérée comme la première référence aux ruines de Tell el-Far'a et à son contexte géographique. Il évoque le tell, la tour et la source d'eau d'el-Far'a dans les termes suivants:

[...] je gravis à pied, à deux heures dix minutes, une belle colline qui s'élève, à une faible distance au sud, à la hauteur d'environ 45 mètres au-dessus de la vallée. Les pentes et le sommet sont actuellement cultivés ; mais, au milieu des fleurs, des blés et des herbes qui le couvrent, on heurte de nombreuses pierres de toutes grandeurs, restes de constructions renversées de fond en comble. Les ruines s'étendent au bas de ce tell, appelé Tell el-Fera'a, jusque sur les bords de l'oued. Là des musulmans avaient construit un petit sanctuaire avec 
des blocs antiques ; il est lui-même, sauf quelques assises inférieures, presque entièrement démoli. [...]. À son extrémité orientale, on remarque, sur son point culminant qui surplombe et commande la source de l'oued, une grande tour carrée mesurant vingt pas sur chaque face [...]. Toutes ces ruines, tant celles qui couvrent le Tell Fera'a que celles qui abondent sur la colline opposée du Bordj el-Fera'a et dans la vallée qui les sépare, témoignent qu'il devait se trouver jadis à la source même de l'A'in el-Fera'a une ville de quelque importance, dont la position était, du reste, très-avantageuse [...]. Au Ras el-A'in Fera'a, au contraire, la source est l'une des plus belles et des plus importantes de la Palestine, et elle a dû, de bonne heure, attirer et fixer près d'elle un centre de population. (Guérin, I874: 258-260).

Quelques années plus tard, en 1874, la région a été visitée par les militaires britanniques $\mathrm{C}$. R. Conder et H. H. Kitchener. Les résultats de cette exploration ont été publiés dans leur ouvrage The Survey of Western Palestine. Les auteurs se réfèrent à l'hydrographie de la zone ("Wady Fârah » et " Râs el Fârah ») et aux vestiges de la tour ("Burj el Fârah »), qu'ils décrivent comme suit : «A square tower of small size, on a knoll, probably built as a guard-house, and not older than Saracenic times ». (Conder et Kitchener, 1882: 223 et 234). Par contre, ils n'apportent rien de nouveau sur Tell el-Far'a, si ce n'est le situer sur une carte topographique (feuille XII) et traduire en anglais la description faite par V. Guérin (Conder et Kitchener, I882: 234-235).

Dans une intense prospection sur le terrain dans la région au nord-est de Naplouse, à la recherche de la ville biblique de Tirza, en I930, l'orientaliste nord-américain W. F. Albright a visité et exploré Tell el-Far'a, qu'il a décrit comme une colline de $300 \mathrm{~m}$ sur $200 \mathrm{~m}$ de dimensions similaires à $\mathrm{Me}$ giddo et correspondant à la moitié de Jéricho et Tell Beit Mirsim (Albright, I93r: 245). Il a décrit le site de la façon suivante :

Little masonry is visible. Shord exposed sections of a megalithic city-wall appear at intervals along the edge of the mound, specially in the north, and

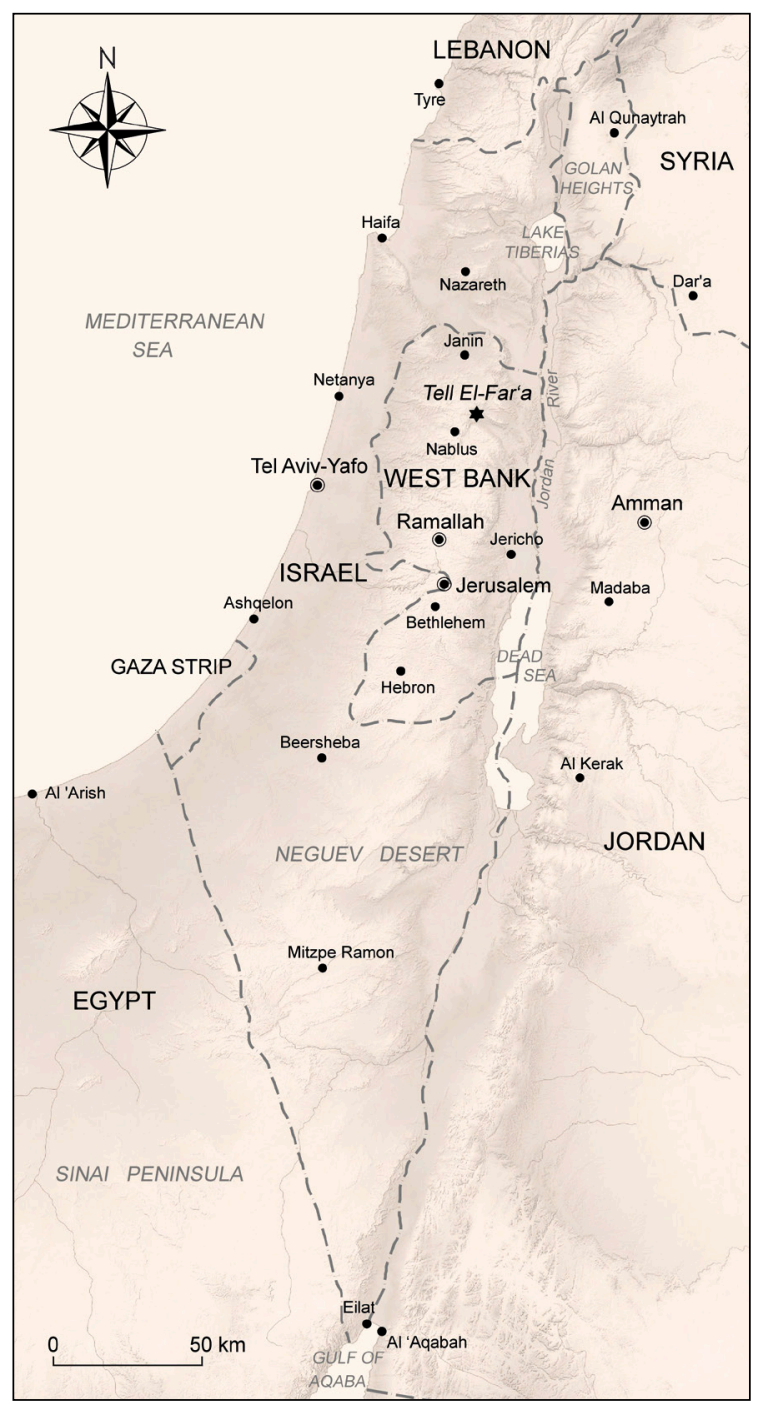

Figure 1. Carte de situation de Tell el-Far'a, Palestine (J.G. Gómez, TEF)

Figure 1. Map of situation of Tell el-Far'a, Palestine (J.G. Gómez, TEF)

evidently mark the line of Bronze Age fortification. The acropolis was sitauted at the western end, which is the highest and most easily defended part of the mound, as well as the part which lies nearest the spring - a most important consideration [...]. This site is large, well-situated, and easily defended; it was occupied during the Bronze and early Iron Ages [...]. From the strategic point of view, Tell el-Fâr'ah was a much better site for the capital of Israel than was Samaria [...]. (Albright, I93I: 246).

À partir de l'étude de la céramique, il a conclu que Tell el-Far'a ("Mound of Elevated Ridge ») a été abandonné au IX ${ }^{\mathrm{e}}$ siècle av. J.-C. Selon lui, il est 
le meilleur candidat, en raison de son emplacement et de ses caractéristiques, pour être l'ancienne ville de Tirza (Albright, I931: 246-247 et 251).

N. Glueck, en 1940, dans le cadre de ses explorations dans la région orientale de la Palestine, a visité plusieurs sites du wadi el-Far'a, dont Tell Miska, situé à $8 \mathrm{~km}$ de Tell el-Far'a. Dans la publication ultérieure, l'archéologue nord-américain a daté ce site de la période chalcolithique en s'appuyant sur des parallèles typologiques avec la poterie de Far'a (Glueck, 1951: 422).

C'est en I946 que Tell el-Far'a a été choisi par le père Roland de Vaux, directeur de l'École Biblique et Archéologique Française (EBAF) de Jérusalem, pour démarrer un grand projet de fouilles avec l'autorisation du Service jordanien des antiquités. Entre le $\mathrm{I}^{\mathrm{er}}$ juin 1946 et le 22 octobre I96o, neuf campagnes de fouilles ont été réalisées au total. Les rapports avec les résultats provisoires des différentes campagnes ont été publiés régulièrement dans la Revue Biblique (De Vaux et Steve, 1947a ; 1947b ; 1948 ; 1949 ; et De Vaux, 1951a ; 1951b ; I952 ; 1955 ; 1957 ; 1961 ; 1962). Par la suite, après la mort du père De Vaux, en I97I, plusieurs monographies ont paru, consacrées au Bronze moyen et à l'âge du Fer, ainsi qu'à l'étude de la céramique, des scarabées et des sceaux (Chambon, 1984; Mallet, 1973 et 1987 ; Amiet et alii, 1996).

Les travaux réalisés à Tell el-Fara (De Vaux, Miroschedji et Chambon, 1993) ont servi à établir une séquence chronologique formée de sept grandes périodes archéologiques (ca. 8500-600 av. J.-C.), à savoir :

\section{PPNB \\ II. Chalcolithique \\ III. Bronze ancien I \\ IV. Bronze ancien II \\ V. Bronze moyen II \\ VI. Bronze récent \\ VII. Fer I-II (avec 5 sous-périodes)}

Il y a, cependant, un problème majeur concernant la chronologie des sept périodes archéologiques de Tell el-Far'a, établie par Roland de Vaux et son équipe : nous n'avons à notre disposition ni séquence stratigraphique minutieuse, ni dates absolues de celle-ci. L'ensemble du système chronologique a été construit à partir de l'identification des phases architecturales et constructives et de l'étude typologique et comparative de la poterie. Sur cette question, qui affecte principalement l'occupation de l'âge de Fer du site (période VII), A. Chambon (1984: 12) a écrit que :

Ces imprécisions majeures, ainsi que d'autres de moindre portée, nécessitent une reprise minutieuse de la stratigraphie et de l'attribution du matériel, et méritent une présentation synthétique, fondée sur l'étude de quelques points-clé et de coupes schématiques reconstituées.

Récemment, M. Jasmin (2013: 394), dans un article de synthèse sur Tell el-Far'a, a exprimé de nouveau la nécessité de réétudier la stratigraphie du site :

De Vaux's excavations in the 1950s were made without rigorous stratigraphic controls, which has led to many uncertainties regarding the stratigraphic context of objects or architectural remains. Neither the first reports in 1950 os and I96os nor the final publications in the I980s solve all the stratigraphic problems. [...]. The reappraisals of Tell el-Far'ah's stratigraphy and history have led to a thorough revision of the Iron-II site's chronology and its relation with the biblical account.

Après la fin des campagnes dirigées par le père De Vaux, en octobre I96o, le site de Tell el-Far'a a vécu une phase d'oubli et d'abandon en ce qui concerne les travaux de terrain, qui va se prolonger pendant cinquante-sept ans. Au cours de cette période, les recherches réalisées ont concerné soit l'étude des matériaux archéologiques et des vestiges architecturaux, soit la réalisation de diverses prospections dans la région environnante du site, en particulier du wadi el-Far'a.

Les études des objets trouvés lors de fouilles de l'EBAF à Tell el-Far'a concernent surtout les céramiques des niveaux du Bronze ancien (Huot, 1967; Miroschedji, 1976 ; Charloux, 2005-2006 ; Medeghini et alii, 20I9) et de l'âge du Fer (Herzog et Singer-Avitz, 2006 ; Kleiman, 2018) dans le but d'avancer dans la connaissance de la typologie, de la chronologie et de la technologie. L'industrie lithique 
des différentes périodes a été étudiée par le préhistorien espagnol J. A. Fernández-Tresguerres (1987). Des « maquettes architecturales » du Fer II et une statuette en bronze et argent ont fait l'objet de diverses recherches (Miroschedji, 200I ; Picard, 1958). Enfin, parmi les études sur l'urbanisme, on doit souligner celles menées par Z. Herzog (1997) et P. de Miroschedji (20I3) sur la période du Bronze ancien II au Fer IIB, et par A. Burke (2008: 257-258) sur les remparts du Bronze moyen II.

Durantl'été 1962, les biblistes allemands S. Kappus et $\mathrm{R}$. Knierim ont effectué une prospection sélective dans le wadi el-Far'a, où ils ont identifié onze sites de différentes périodes historiques (Kappus, 1966 ; Knierim, 1969). À la suite de la guerre des Six jours, une autre prospection archéologique a été organisée entre l'été 1967 et l'hiver 1968 dans les régions de Juda, de Samarie et du Golan par des archéologues israéliens. La publication de cette recherche, en hébreu, contient l'étude de plusieurs sites du wadi el-Far'a (Kochavi éd., I972). Quelques années plus tard, un autre archéologue israélien, Z. Ilan, dans son étude de la vallée du Jourdain et du désert de Samarie, s'est rendu dans la région de Tell el-Fara (Ilan, 1973: 365-369).

Entre 1980 et 1994, A. Zertal, professeur d'archéologie à l'Université de Haïfa, a lancé son projet "The Manasseh Hill Country Survey ", qui comprenait une étude systématique du wadi el-Far'a, où il a identifié 47 sites archéologiques dont la chronologie va du néolithique à la période islamique, et même postérieure à cette dernière. D'après son étude, qui a été taxée de fondamentaliste (Marcus, 2000: 98), une occupation importante du territoire s'observe au Bronze ancien I, au Fer II et la période byzantine. Par contre, les sites du Bronze moyen sont rares (Zertal, 2008: 44-45 et 419-504). Quant à Tell el-Far'a, il a proposé la séquence chronologique suivante à partir de l'étude des tessons collectés : Néolithique, Chalcolithique, Bronze ancien I-IV, Bronze moyen I et IIB, Bronze récent I-III, Fer IC, II et III, et Perse (Zertal, 2008: 42I).

Entre 2001 et 2002, K. Abdulfattah, de l'Université de Birzeit, et B. de Vries, du Calvin College (Michigan), ont coordonné un projet de coopération internationale pour réaliser une évaluation environnementale du wadi el-Far'a (Abdulfattah et De
Vries eds., 2005). L'étude du wadi comprenait également un inventaire du patrimoine culturel dans le but de développer une base de données, comprenant les IIo sites archéologiques documentés de différentes périodes historiques. D'après l'avis des auteurs de l'étude :

In general, the area's material cultural heritage has suffered due to lack of or poor site management. The team found that a large number of the sites in the Wadi el-Far' have been plundered and partially damaged by ilegal excavactions and other means of human destructions. (Dababsa et alii, 2005: 83).

Ils proposent que de nouvelles études soient menées afin de valoriser du point de vue touristique les sites de Tell el-Far'a et Burj el-Far'a (Dababsa et alii, 2005: 85).

T. Bryce (2012: 240) dans un livre récent sur les peuples et les sites de l'Orient ancien a très bien synthétisé la valeur historique de Tell el-Far'a pour la connaissance du Levant antique :

Tell el-Farah is none the less ranked alongside Megiddo and Hazor in terms of the importance of the information it provides about northern Canaan and the hill country.

Cette importance justifie les nouveaux travaux entamés sur le site en 2oI7 par une équipe internationale.

\section{3. À propos du nom ancien de Tell el-Far'a}

V. Guérin a été le premier à proposer un nom ancien aux ruines archéologiques situées près de la source d'Aïn el-Far'a, qu'il avait attribué à une ville importante :

Je ne serais pas éloigné de penser que cette ville fut celle d'E'n-Tappouah. [...]. Comme le mot Tappouah, en hébreu, signifie pomme et, par extension, d'autres espèces de fruits, il est permis de penser, ce qui est, du reste, très-présumable, que les anciens avaient dû profiter des eaux intarissables de l'A'in el-Fera'a pour planter alentour 
de beaux vergers ; de là, le nom d'E'n Tappouah donné, selon ma supposition, à la ville qui s'élevait en cet endroit. (Guérin, I874: 259-260).

La Bible place la ville d'En-Tappúah à la frontière de Manassé et d'Éphraïm (Js I7, 7-8). Ce toponyme ancien a été identifié avec Tell Abu Zarad, au sud de Sichem (Abel, 1938: 58 ; Nigro et alii, 2015).

D'autres noms anciens ont été proposés pour Tell el-Far'a. A. Alt (1927: 36-38 et 1932: 40-44) a suggéré une identification avec Ophra, du clan Abièzer (Jg 6, II et 8, 27). F. M. Abel (1938: 268) a souligné la possibilité qu'il s'agisse de l'ancienne Beth-Bara, un toponyme lié au Jourdain $(\mathrm{Jg} 7,24)$. Mais le nom antique qui a suscité le plus de débats est celui de Tirza, une ville citée dans l'Ancien Testament (I R I4-I6 ; ${ }_{2} \mathrm{R}_{15}$; Js I2, 24 ; etc.). En I84I, E. Robinson (I84I: 158) a proposé, en raison de sa similitude, que Talluza, à moins de $5 \mathrm{~km}$ au sud-ouest de Tell el-Far'a, soit la ville biblique de Tirza. W. F. Albright (I93I: 24I-25I) a été le premier chercheur à proposer l'identification de Tell el-Far'a avec Tirza, où le roi Jéroboam I Ir a placé sa résidence officielle jusqu'à ce qu'Omri ait déplacé sa cour dans la ville de Samarie vers 880 av. J.-C. (Briend, 1996: 9-I4). Le père De Vaux (1956: I35-I40 ; I967; 1976: 395), malgré ses doutes initiaux, a accepté finalement de localiser l'ancienne ville de Tirza à Tell el-Far'a (Stratum 3 ou période VIIb).

D'après le récit biblique, le roi Jéroboam $\mathrm{I}^{\mathrm{er}} \mathrm{a}$ d'abord fortifié la ville de Sichem, où il résidait, puis il est parti pour construire Penouël (I R I2, 25). Sichem est Tell Balata, un parc archéologique situé aujourd'hui à la périphérie de Naplouse. Penouël pourrait être Tell ed-Dhahab, dans le wadi Zarqa, en Transjordanie (Pola et alii, 20I6). La ville de Tirza est mentionnée dix-sept fois dans la Bible hébraïque. Dans I R I4, I7, on dit que : « La femme de Jéroboam se leva et partit. Elle arriva à Tirza ». De cette citation, on peut déduire que Tirza était la résidence de Jéroboam, le premier monarque du royaume d'Israël. Elle était donc la ville où se trouvait sa maison, c'est-à-dire la capitale du royaume du nord dans le dernier quart du $\mathrm{x}^{\mathrm{e}}$ siècle av. J.-C. (Laato, 2015). Pendant le règne de Basha (ca. 908-885 av. J.-C.) il n'y a aucun doute, d'après la Bible, sur le fait que Tirza soit la capitale : «La troisième année d'Asa, roi de
Juda, Basha, fils d'Ahija, régna sur tout Israël à Tirza. Il regna vingt-quatre ans. » ( $\mathrm{R}$ 15, 33). Tirza a été le siège des sept premiers souverains du royaume du nord, pendant une période d'environ 45 ans. Dans la sixième année (vers 880 av. J.-C.) de son règne, Omri a déplacé la capitale à Samarie ( $\mathrm{R} \mathrm{I6,} \mathrm{24).}$

En dehors de la Bible, Tirza apparaît dans un texte du grand temple d'Amon à Karnak, dans lequel il y a une liste des villes conquises par le pharaon égyptien Sheshonk I, fondateur de la XXIIe dynastie, lors de sa campagne à travers la Palestine. Dans son expédition de Gaza vers le nord, il est arrivé à la région de la vallée moyenne du Jourdain. Malheureusement, le nom de Tirza n'est pas entièrement conservé dans l'inscription (Lipinski, 20I8: 60). Si la restitution proposée du toponyme est certaine, sa présence dans ce texte égyptien confirme, certes, l'existence de la ville au $\mathrm{X}^{\mathrm{e}}$ siècle av. J.-C. (Ahituv, I984: 20 et I90 ; Kitchen, 1973: 432-447). Cependant, il ne fournit pas de données sur sa localisation géographique. Alors, où était située l'ancienne ville de Tirza ?

Le nom féminin Tirza vient d'une racine hébraïque, qui fait référence à " plaisir » et « beauté ». Ces qualificatifs étaient traditionnellement liés à la nature de l'environnement géographique dans lequel on pensait que cette ville antique pouvait être située : un lieu d'accès facile à la vallée du Jourdain, ce qui a rendu possible l'occupation humaine. Ces mêmes caractéristiques sont également attribuables à Tell el-Far'a.

De même, on pense que Tirza devait être une ville vraiment belle, puisque Salomon l'a comparé à la beauté de sa bien-aimée $(\mathrm{Ct} 6,4)$. Une approche de la signification de la racine arabe $f r^{4}$ et du lexique qui en dérive ne permet pas d'affirmer que Tirza est Tell el-Far'a, mais on peut conclure que les passages de Tirza cités dans l'Ancien Testament et les nombreuses significations liées à la racine arabe $f r^{\prime}$, d'où vient el-Fara, sont concordants. Cela nous permet d'entrevoir une certaine relation entre les deux termes (Montero Fenollós et alii, 2020b: II2-IIg).

L'Ancien Testament ne donne aucune indication sur la situation géographique de Tirza. Quatre textes bibliques ( $\mathrm{Nb} 26,33 ; 27, \mathrm{I} ; 36$, Ir et $\mathrm{Js} \mathrm{I} 7,3$ ) mentionnent Tirza comme l'une des cinq filles de Celophehad, qui ont été interprétées comme cinq localités du territoire de la tribu de Manassé. De cela, 
on a déduit que la ville serait située sur le territoire de cette tribu (située au nord de la Cisjordanie). D'après les ostraca de Samarie (Lemaire, I977), des documents écrits en paléo-hébreu du viII ${ }^{\mathrm{e}}$ siècle av. J.-C., il est possible de situer Tirza au nord-est de Sichem (Briend, I996: I2-I4). Tell el-Far'a, à ıo km à vol d'oiseau de Sichem, est un bon candidat.

En faveur de cette identification, des preuves archéologiques ont été avancées également à partir de la comparaison des séquences chronologiques de Tell el-Far'a et de Samarie. La rupture observée entre les deux séquences archéologiques est étayée par le fait que parmi la céramique de l'âge du Fer à Tell el-Far'a, les vases typiques des premières périodes de Samarie, fondée par Omri ca. 880 av. J.-C., sont absents. Par contre, en Samarie, la poterie de la période précédente, trouvée à Tell el-Far'a n'a pas été attestée (Kenyon, 1979: 258-259). De même, l'existence à Tell el-Far'a d'un grand bâtiment (dit " inachevé » par ses fouilleurs) a été interprétée, en vertu du texte biblique, comme la preuve matérielle de l'abandon de la ville au temps du roi Omri (De Vaux, I976: 403).

La localisation de Tirza à Tell el-Far'a est l'hypothèse communément acceptée par l'historiographie actuelle sur l'ancien Israël (Lipinski, 20I8: 56). A. Zertal (2008: III) a considéré cette identification comme la plus probable et rejette la proposition de localiser Tirza dans la basse Galilée (Sapir, 199I). Pour I. Finkelstein (2013: I09-IIO) :

Tirça a été identifiée à Tell el-Far'ah dans les Haute Terres au nord-est de Sichem [...]. Tout cela fait de Tell el-Far'ah (le site de Tirça) l'élément clé de l'étude des débuts du Royaume du Nord.

Mais il a introduit une nouveauté chronologique. Pour lui, la ville de Tirza correspondrait à la période VIIa et non à la période VIIb du site, comme cela est traditionnellement signalé (Finkelstein, 20I2). De son côté, W. Dever (200I: I42), qui a accepté l'identification entre Tirza et Tell el-Far'a, a défendu une autre hypothèse : la ville de la période VIIa, qu'il date du $\mathrm{x}^{\mathrm{e}}$ siècle av. J.-C. en raison de la céramique, aurait pu être un centre administratif dans le nord au service du roi Salomon.
Finalement, il faut noter que les principaux atlas sur le Proche-Orient antique et le monde ancien placent sans hésitations Tirza à Tell el-Far'a (Kellermann et alii, I992 ; Jasmin, 2020: I2O ; Wittke, Olshausen et Szydlak eds., 20I2: 45). Cependant, il est juste de reconnaitre qu'en l'absence de documentation épigraphique, qui fournirait des preuves irréfutables, cette identification, même si elle est très probable, ne reste qu'une hypothèse.

\section{Le nouveau projet archéologique}

Après environ 60 ans d'oubli, il est clair que Tell el-Far'a méritait d'être réétudié par de nouvelles méthodes de travail afin de faire progresser la compréhension de l'âge du Bronze et du Fer dans cette région clé du Proche-Orient ancien. Pour cette raison, en 2017 , un projet archéologique international a été lancé par l'Université de La Corogne, l'Université NOVA de Lisbonne et le Ministère du Tourisme et des Antiquités de Palestine avec l'objectif central de mener des fouilles arhéologiques à Tell-el-Fará.

Ces dernières années, Tell el-Far'a a subi d'importantes transformations liées à l'activité des habitants du wadi el-Far'a. Actuellement, la propriété du site est partagée entre quatre propriétaires privés et le gouvernement palestinien. Une partie importante du secteur privé du site est affectée par différentes activités agricoles, en particulier par l'exploitation des oliviers. Les principales zones libres de travaux agricoles sont situées dans le secteur oriental et surtout dans la partie ouest du tell. C'est dans ce dernier secteur, appartenant au domaine public, que les archéologues français ont concentré l'essentiel de leurs travaux de fouille jusqu'en rg6o. Dans cette partie du site, d'importants vestiges architecturaux (maisons, remparts, portes, etc.) sont conservés. L'état de conservation de ceux-ci est bon, en général, à l'exception de la porte monumentale en brique crue du Bronze ancien (De Vaux, I976: 400), fortement affectée par l'érosion.

En 20I7, un nouveau plan de Tell el-Far'a a été élaboré pour nous aider à comprendre l'évolution topographique du site entre 1946 (date à laquelle le premier plan a été réalisé par l'équipe du père De Vaux) et nos jours. Un plan du tell et de ses environs 
a été obtenu : un modèle $3 \mathrm{D}$ (orthophotoplan) et un modèle conventionnel avec des courbes de niveau. Dans les deux cas, tant les différentes utilisations actuelles du site que la localisation des déblais lors des fouilles de l'EBAF, qui ont altéré la morphologie d'origine du site, ont été analysées, notamment dans la périphérie ouest du site.

Le but des recherches du nouveau projet est l'étude de la grande période qui va de la naissance des premières villes fortifiées en Palestine (vers 3000 av. J.-C.) jusqu'à l'arrivée de l'Empire assyrien dans la région (vers 720 av. J.-C.), c'est-à-dire, entre le Bronze ancien II et l'âge du Fer IIC. Bien que le projet soit encore dans une phase initiale, les nouveaux travaux de fouilles archéologiques à Tell el-Far'a, réalisés lors des campagnes 20I7, 2018 et 20I9, ont fourni de nouvelles données sur l'occupation à l'âge du Fer II (Montero Fenollós et alii, 2019; 2020a et 2020b).

Les travaux de fouille réalisés à ce jour se sont concentrés sur l'ancien chantier II (I950-I960), dans le secteur occidental du site (figure 2), où une zone (dite « $\mathrm{A}$ ») a été ouverte et organisée en quatre sous-secteurs : A $(6 \times 5 \mathrm{~m}), \mathrm{AI}_{\mathrm{I}}(7,30 \times 5 \mathrm{~m}), \mathrm{A}_{2}(3 \times 5 \mathrm{~m})$ et $\mathrm{A}_{3}(9,70 \times 4 \mathrm{~m}) . \mathrm{La}$ fouille a permis d'identifier différents éléments de construction, qui correspondent à trois phases chronologiques de l'âge du Fer, entre le $\mathrm{x}^{\mathrm{e}}$ et le viII ${ }^{\mathrm{e}}$ siècle av. J.-C. Ces travaux ont été suivis d'une prospection sélective dans le wadi el-Fara, et ses environs, afin de connaître le peuplement régional et pouvoir reconstruire la zone d'influence politique et économique des anciens habitants de Tell el-Fara entre le Bronze ancien et l'âge du Fer (Montero Fenollós et alii, 2019; 2020a et 2020b). La recherche de terrain développée au cours des trois premières campagnes a été complétée par une étude sur la toponymie de Tell el-Far'a et sa région (Montero Fenollós et alii, 2020b: II2-II9), et par un projet pilote de restauration et de mise en valeur d'une maison de l'âge du Fer (no 327), fouillée en 1951 par l'équipe de l'EBAF (Chambon, 1984: I68 ; Pardo et alii, 2021: 139-I49).

\section{La « Maison A » de Tell el-Far‘a}

Dans les campagnes 20I7-20I9, une série d'unités constructives (UC), qui composent un bâtiment rectangulaire de 6,70 × 8,90 m, ont été identifiées. Il s'agit d'une unité d'habitat, que nous avons appelée « Maison A » (figures 3 et 4). Les UC attestées lors de la fouille sont les suivantes :

- UC.Io3 : mur de moellons disposés transversalement, qui conserve trois assises de pierres. Longueur : 6,70 $\mathrm{m}$; largeur : o,35 $\mathrm{m}$; hauteur préservée : o,55 $\mathrm{m}$.

- UC.IO4 : pavement en pierre. Longueur : 2,IO $\mathrm{m}$; largeur : I,90 m.

- UC.IO5 : muret à trois piliers en pierre, parallèle à UC.Io3.

- UC.ro6 : mur de pierre, qui conserve deux assises. Fait retour d'angle avec UC.ro3 et UC.ro5. Longueur : 2,00 $\mathrm{m}$.

- UC.III : mur de moellons disposés transversalement et calés par des petites pierres. Longueur : 6,35 $\mathrm{m}$; largeur : ०,45-०,35 $\mathrm{m}$; hauteur préservée de quatre assises : $0,90-0,75 \mathrm{~m}$.

- UC.II2 : mur ayant les mêmes caractéristiques que le mur UC.III, avec lequel il fait retour d'angle. Longueur : 8,90 m ; largeur : o,42-0,38 m ; hauteur préservée : ०,60-0,50 $\mathrm{m}$. Il a une porte de I,25 $\mathrm{m}$ de large qui donne vers la rue UC.Ir8.

- UC.II3 : mur en pierre construit avec deux techniques : parements de moellons disposés transversalement et rangées de moellons avec remplissage interne de petites pierres. Longueur : 8,60 m ; largeur : $0,40-0,45 \mathrm{~m}$.

- UC.II4 : pavement en pierres de différentes tailles. Longueur : 2,I0 $\mathrm{m}$; largeur excavée : $2 \mathrm{~m}$.

- UC.Ir6 : restes de deux tannours partiellement conservés. Diamètre : $0,50 \mathrm{~m}$; hauteur préservée : o, I7-0,33 m.

- UC.II8 : rue de terre avec de petites pierres. Longueur fouillée : Io,60 m ; largeur : 1,90-1,83 m.

- UC.II9 : pavement en pierres de différentes tailles. Longueur : 3,60 m ; largeur : 2,00 m.

- UC.I20 : mur de moellons disposés transversalement et calés par des petites pierres. Longueur : 5,70 $\mathrm{m}$; largeur : ०,40-0,45 $\mathrm{m}$; hauteur préservée : $0,73 \mathrm{~m}$. Il a une porte de $72 \mathrm{~cm}$ de large dans laquelle la crapaudine a été trouvée in situ.

- UC.122 : fragment d'un tannour qui était sur un cercle de petites pierres. Il est situé à l'extérieur du mur UC.Io3. 


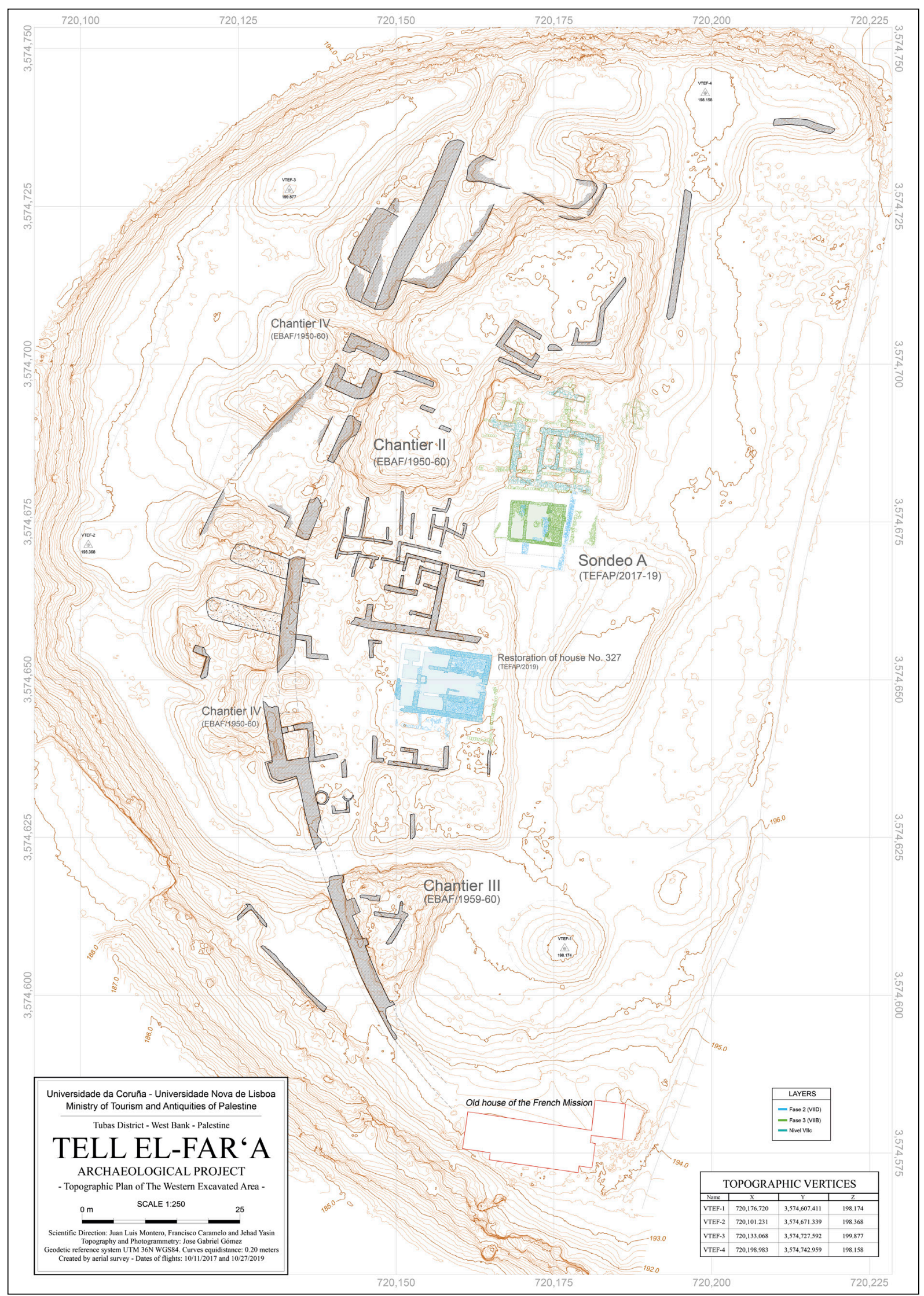

Figure 2. Plan topographique du secteur occidental du site (J.G. Gómez, TEF)

Figure 2. Topographic plan of the western sector of the site (J.G. Gómez, TEF) 


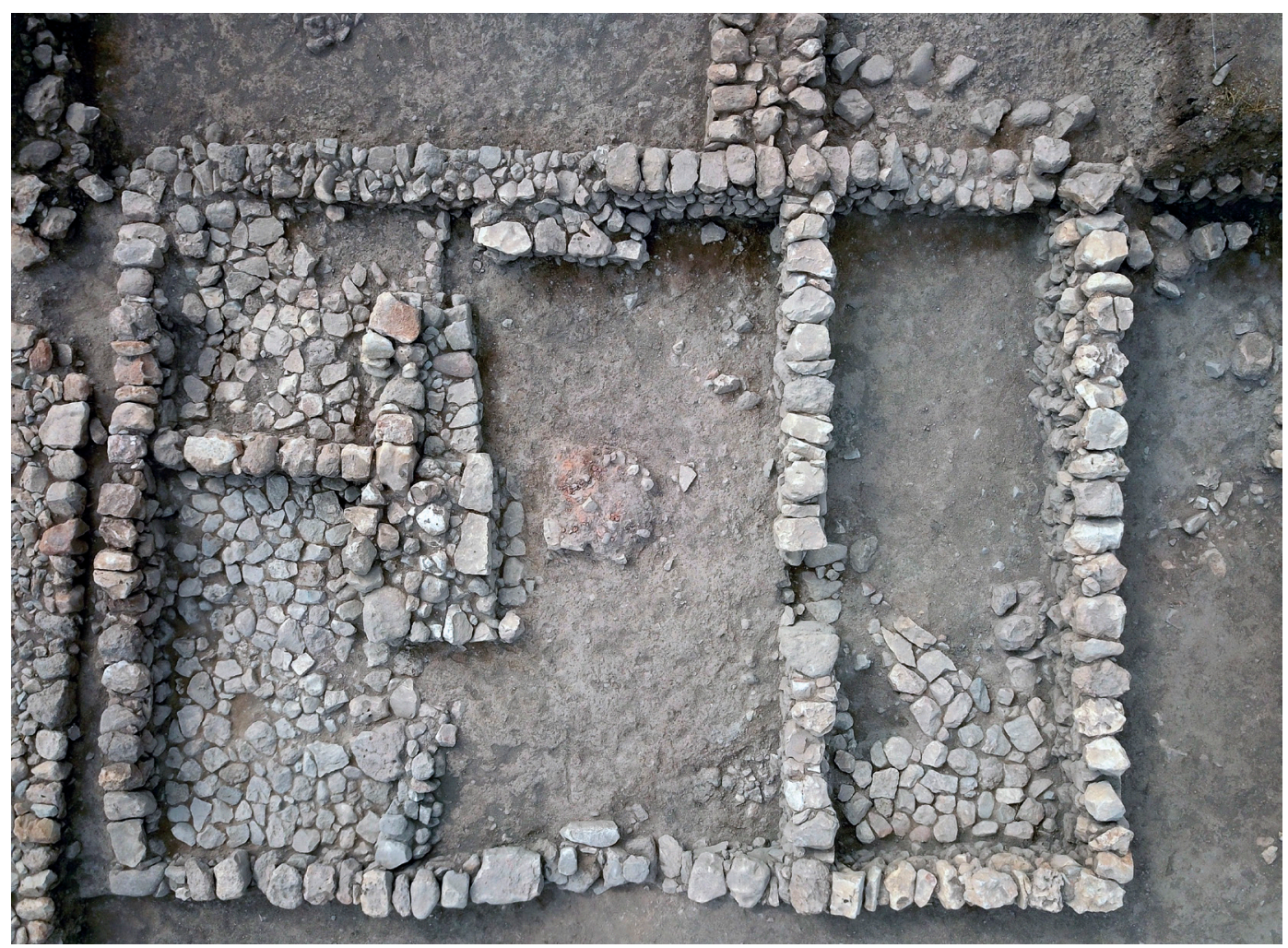

Figure 3. Vue aérienne de la « Maison A » (J.G. Gómez, TEF)

Figure 3. Aerial view of "House A" (J.G. Gómez, TEF)

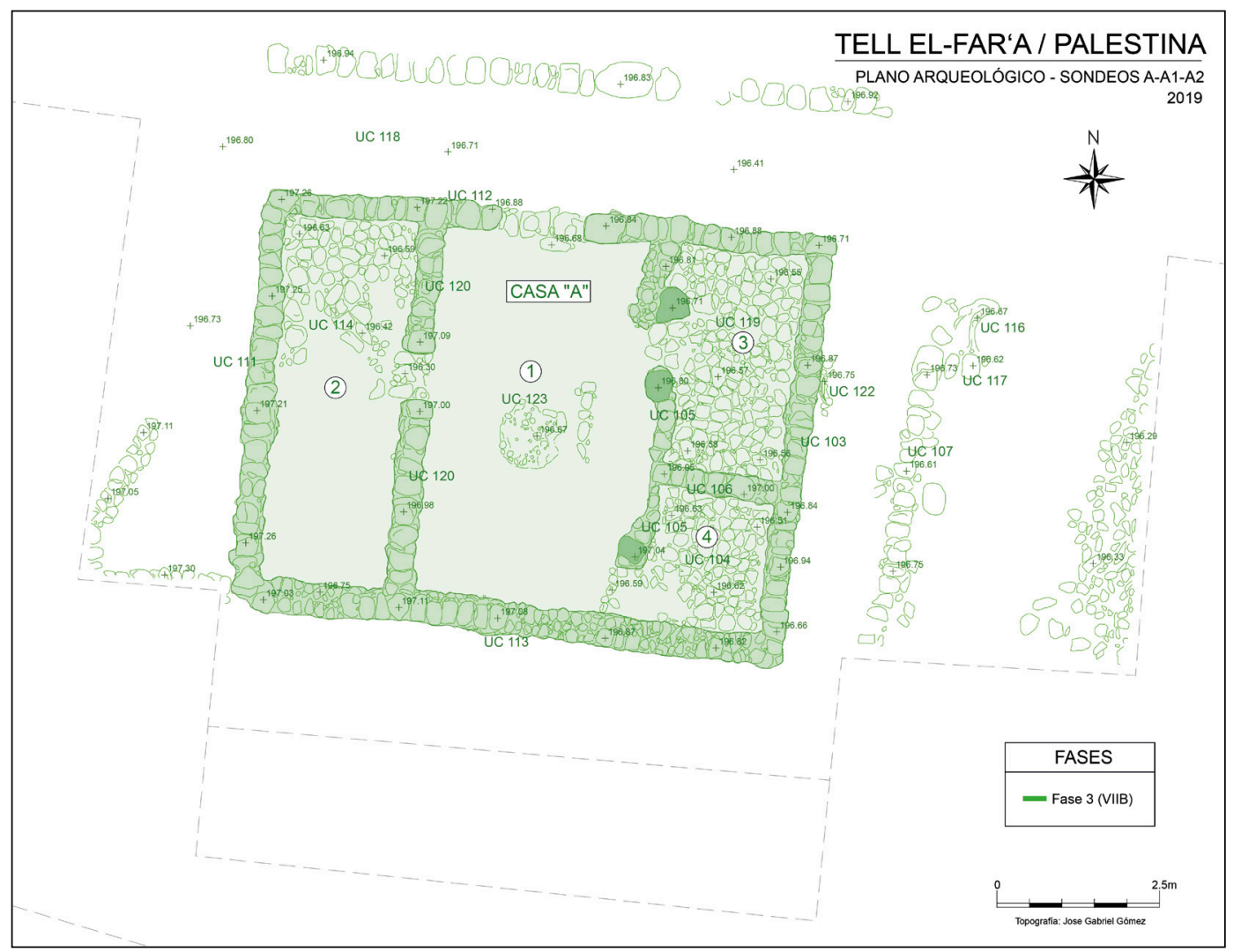

Figure 4. Plan de la « Maison A » (J.G. Gómez, TEF)

Figure 4. Plan of "House A" (J.G. Gómez, TEF) 


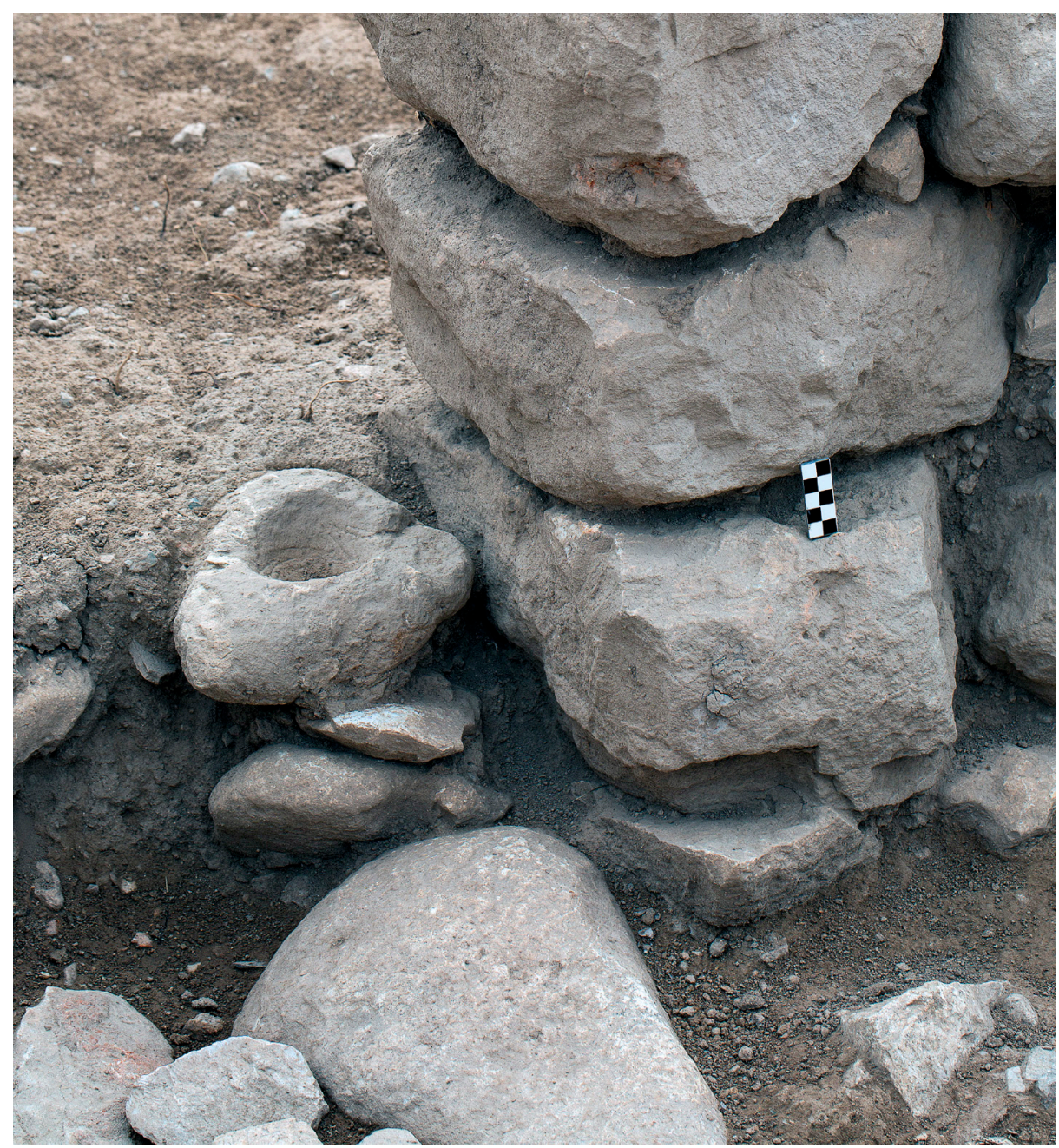

Figure 5. Crapaudine in situ de la « Maison A » (J. Pardo, TEF)

Figure 5. Stone door socket in situ of "House A" (J. Pardo, TEF)

- UC.I23 : vestiges d'un tannour situé au centre de la pièce $\mathrm{n}^{\mathrm{o}} \mathrm{I}$, qui était construit sur un cercle de petites pierres. Diamètre : $0,70 \mathrm{~m}$.

- UC.I27 : sol en terre battue délimité par les murs UC.II2, I20, II3 et 105. Longueur : 5,60 m ; largeur : $3,30 \mathrm{~m}$.

Toutes ces unités constructives décrites font partie de la «Maison A », de $40 \mathrm{~m}^{2}$ de surface environ, qui est composée de quatre pièces différentes :

- La pièce $\mathrm{n}^{\mathrm{o}}$ I $(5,60$ × 3,30 m), ou espace central, avait une porte qui donnait vers la rue UC.II8. Le sol était de terre battue et il y avait un tannour au centre.

- La pièce $\mathrm{n}^{\mathrm{O}} 2(5,70 \times 2 \mathrm{~m})$ avait un sol pavé de pierres dans le secteur nord, tandis que le reste était en terre battue. L'accès à la pièce se faisait par une porte, qui conservait in situ une crapaudine en pierre (avec un trou circulaire de $10 \mathrm{~cm}$ de diamètre) pour soutenir le pivot du vantail. C'est la première crapaudine (figure 5) qui a été mise au jour pour une maison de la période VIIb de Tell el-Far'a (Chambon, I984: 30).

- La pièce $\mathrm{n}^{\mathrm{o}} 3(3,43 \times \mathrm{I}, 90 \mathrm{~m})$ avait un pavement en pierre et avait, comme élément de séparation par rapport à la pièce I, deux murets construits entre deux piliers en pierre dont la base de forme irrégulière est conservée $(55 \times 40 \mathrm{~cm})$. Entre eux, il y avait une porte de $72 \mathrm{~cm}$ de large. Ces murs à piliers représentent l'aspect le plus remarquable des constructions de la période VIIb (Chambon, I984: 29).

- La pièce $\mathrm{n}^{\circ} 4(2, \mathrm{IO} \times \mathrm{I}, 90 \mathrm{~m})$ était pavée de pierres et avait un pilier, qui conservait encore trois pierres 


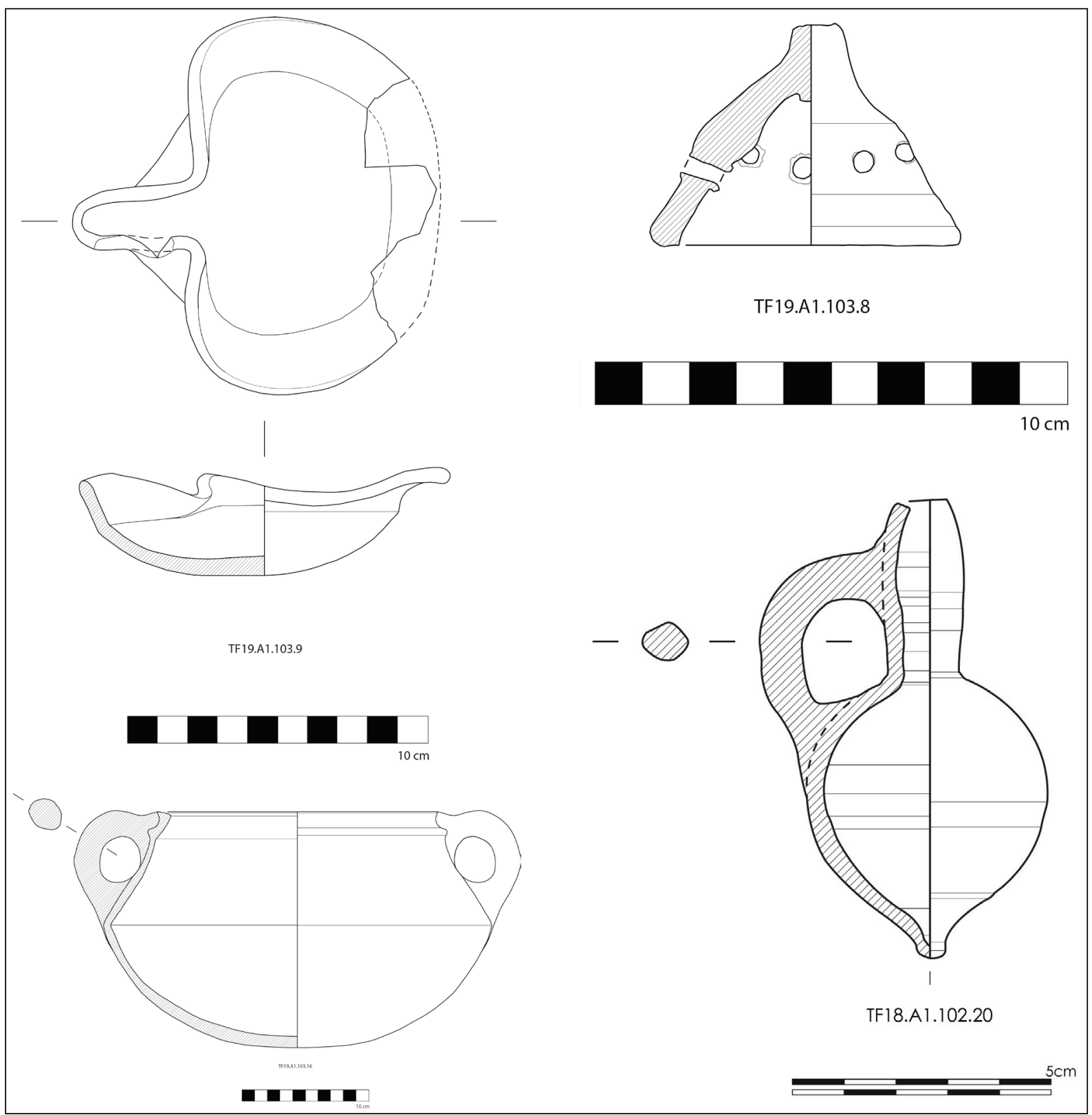

Figure 6. Des céramiques trouvées dans la « Maison A » (A. Bargão et J. Gonçalves Araújo, TEF)

Figure 6. Potteries found at "House A" (A. Bargão and J. Gonçalves Araújo, TEF)

ou tambours en hauteur, de $55 \mathrm{~cm}$ de large. Ce pilier avait un muret de fermeture à sa gauche, et à sa droite une porte de $80 \mathrm{~cm}$ de large, qui donnait sur la pièce $\mathrm{n}^{\mathrm{o}} \mathrm{I}$.

Parmi le mobilier quotidien découvert à la « Maison A », nous mettons en évidence, à titre d'exemple (figures 6 et 7), quelques vases en céramique complets trouvés sur le sol UC.I27 de la pièce $\mathrm{n}^{\circ} \mathrm{I}$ (une lampe à huile, une passoire et une marmite) et sur le sol UC.II4 de la pièce $\mathrm{n}^{\mathrm{o}} 2$ (une cruchette noire) :
- TF.ig.Ar.ro3.9. Lampe fragmentée, mais conservée pratiquement complète. Elle se compose d'une coupelle, faite au tour, qui a un bec très marqué. La paroi a une épaisseur de 0,4 à $0,6 \mathrm{~cm}$ et la couleur de la surface est orange. La base n'est pas plate, mais légèrement arrondie. Plusieurs exemplaires de ce type de lampe ont été attestés pour la période VIIb de Tell el-Far'a (Chambon, I984: pl.59: I-4). Sa production est très courante dans la région palestinienne à partir de l'âge du Fer IIA (Sussman, 2007: 6I-62). 


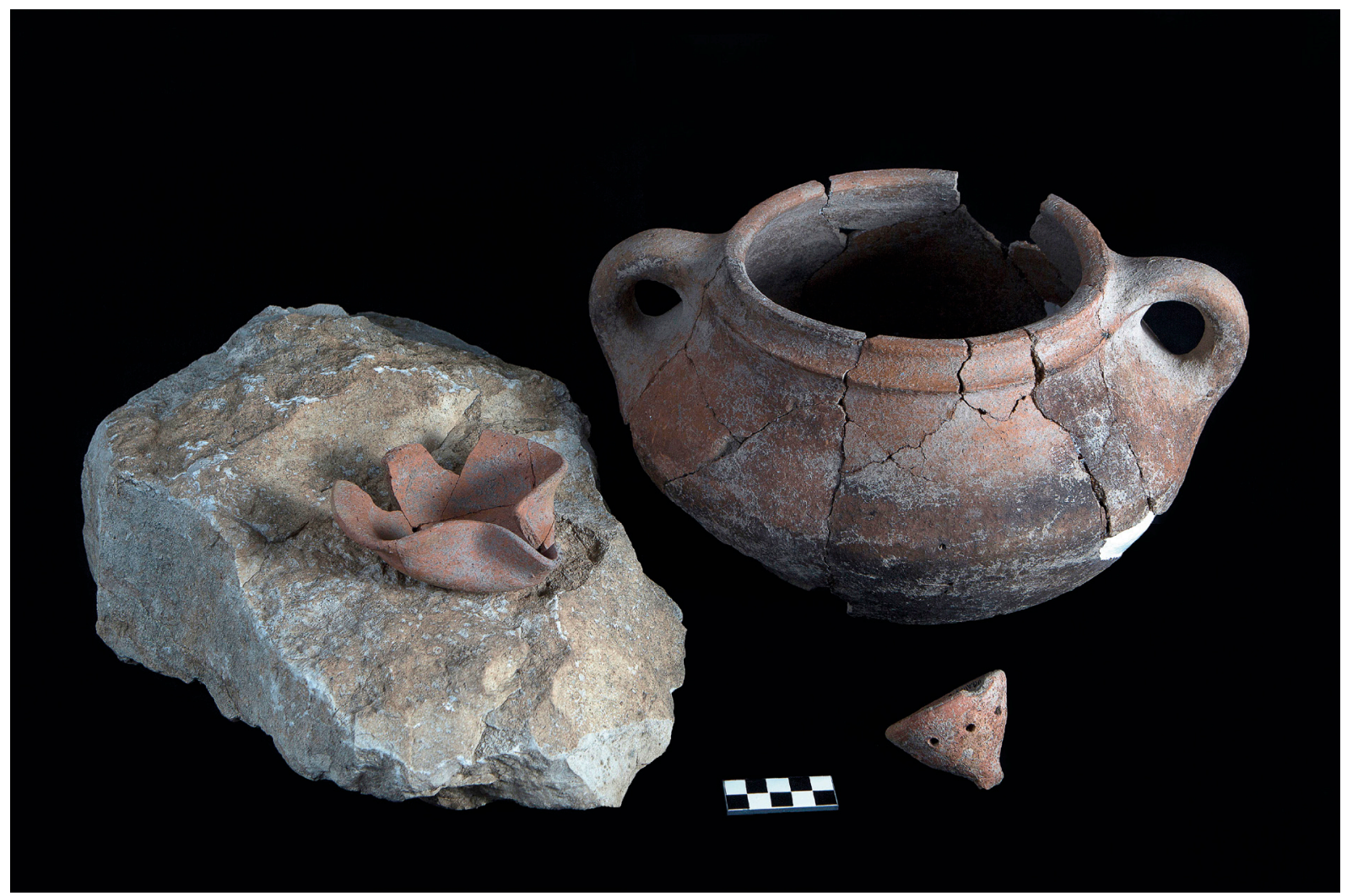

Figure 7. Marmite, lampe et petit vase filtrant de la « Maison A » (J. Pardo, TEF)

Figure 7. Cooking pot, lamp and small perforated cup from "House A" (J. Pardo, TEF)

- TFrg.Ar.Io3.8. Petit vase filtrant ou passoire de $6,5 \mathrm{~cm}$ de diamètre et 0,7 d'épaisseur. La couleur de la surface est brune. Un autre exemplaire en céramique vient de la période VIIb de Tell el-Far'a (Chambon, 1984: 212-213).

- TFig.Ar.ro3.r6. Marmite à feu sans col pourvue de deux anses et corps caréné. La lèvre est formée par un repli vers l'intérieur suivi d'un modelage qui forme un rail circulaire. La paroi a une épaisseur de $0,8 \mathrm{~cm}$, la couleur de la surface est brune et la bouche a un diamètre de $20 \mathrm{~cm}$. Dans les fouilles de Tell el-Far'a, elle est attestée pour la période VIIb (Chambon, 1984: 208, pl. 52: 8). La marmite-pot carénée n'est pas un type exclusif de la région de Samarie, car elle a été retrouvée ailleurs, dans d'autres régions du nord de la Palestine pendant l'âge du Fer II (Tappy, 2015: I9I et 20I ; Ben-Tor et Zarzecki-Peleg, 2015: I40).

- TFr8.Ar.ro2.20. Cruchette noire (figure 8) ou «black juglet » de $9 \mathrm{~cm}$ de hauteur avec un col cylindrique, une bouche légèrement en retrait de $2 \mathrm{~cm}$ de diamètre, un corps globuleux, une anse et un petit appendice à la base. Elle était fortement lustrée. Ce type de cruchette est bien connu dans le nord de la Palestine entre le $\mathrm{x}^{\mathrm{e}}$ et le Ix $\mathrm{x}^{\mathrm{e}}$ siècle av. J.-C. (Tappy, 20I5: I9; Kelley, 20r4: 203). À Tell el-Far'a, il est bien attesté dans la période VIIb (Chambon, I984: 202-204).

Dans le but de réfléchir aux techniques de construction de la «Maison $\mathrm{A}$ » et aux usages des différentes pièces, une hypothèse de reconstruction du bâtiment en $3 \mathrm{D}$ a été élaborée (figures 9 et ro). Du point de vue de la technique constructive, il faut noter que les murs sont entièrement construits en pierre et que la plupart ne sont qu'à un parement de moellons disposés transversalement et calés par des petites pierres. L'irrégularité de la face intérieure et extérieure des murs a formé une surface parfaite pour bien supporter un enduit épais d'argile et de paille hachée, ce qui a contribué à une bonne isolation thermique.

En ce qui concerne la fonction possible de chacune des pièces, la proposition est la suivante : la pièce $\mathrm{n}^{\circ} \mathrm{I}$ était un espace à ciel ouvert équipé d'un 


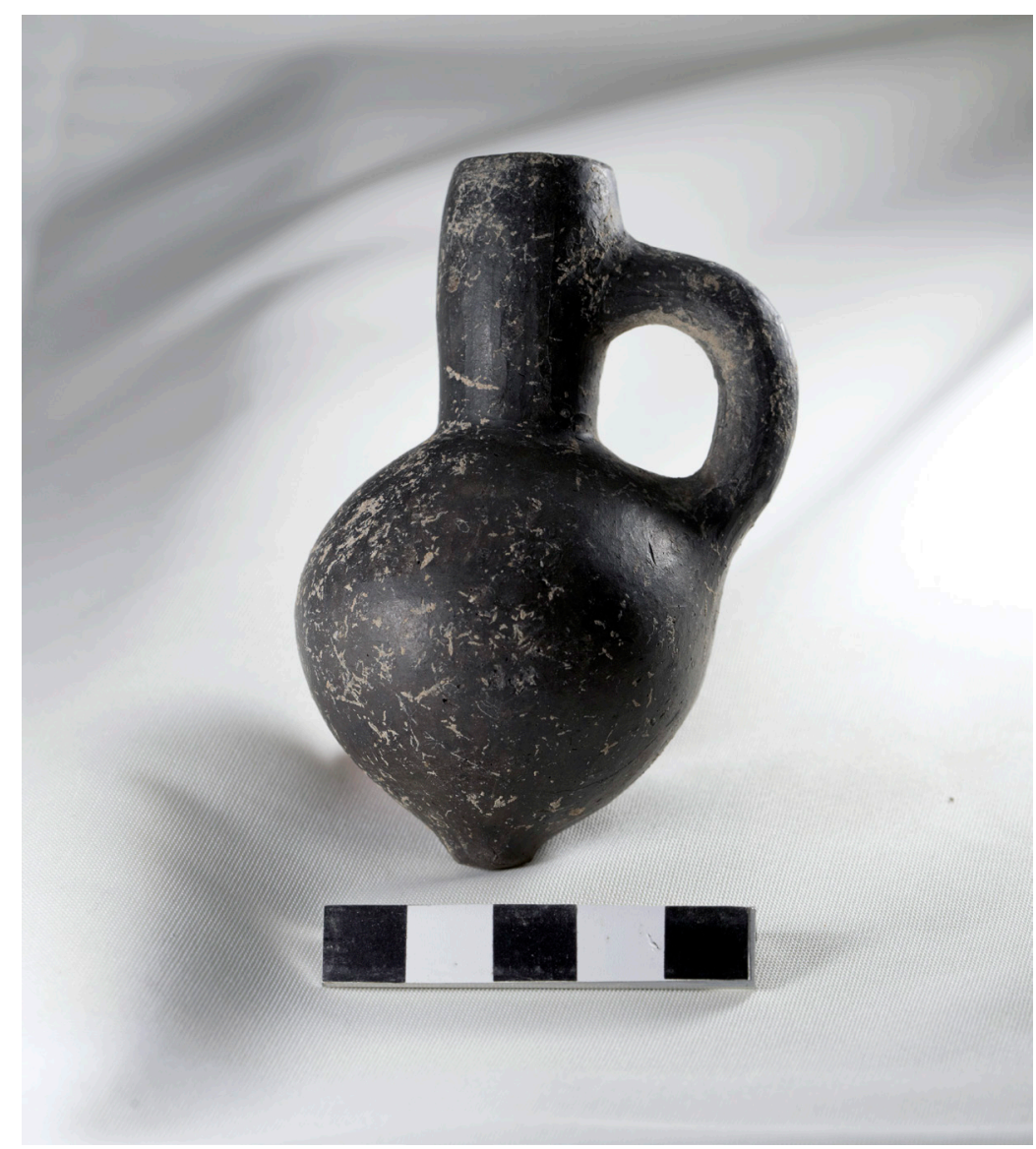

Figure 8. Cruchette noire de la « Maison A » (J. Pardo, TEF)

Figure 8. Black juglet from "House A" (J. Pardo, TEF)

four à pain ou tannour et d'un foyer pour cuisiner. La pièce $\mathrm{n}^{\mathrm{o}} 2$ avait une porte qui pourrait être fermée. C'est, selon toute vraisemblance, la zone réservée à la cellule familiale. Enfin, les pièces $n^{\circ} 3$ et 4 étaient séparées de l'espace central par des murets à piliers qui ne semblent pas avoir été élevés jusqu'à la toiture. Ils permettent une aération aisée des pièces. Les piliers ont servi à supporter, semble-t-il, des poteaux en bois qui soutenaient la toiture. Les deux espaces doivent avoir été utilisés pour garder des animaux domestiques. La fragilité des murs (40 cm de large environ), ainsi que l'absence de traces d'un escalier à l'intérieur, font penser qu'il s'agissait d'un bâtiment simple à nature domestique dépourvu d'étage.

Les fouilles françaises à Tell el-Far'a ont mis au jour plusieurs maisons de la période VIIb, toutes construites avec les mêmes techniques de construction (murs d'un parement de moellons disposés transversalement), mais plus grandes que la
« Maison A ». Le plan général le plus courant de cette période est celui d'une maison composée de cinq ou six pièces qui s'ouvrent sur un espace central rectangulaire avec un sol en terre battue (voir, par exemple, les maisons $n^{\circ}$ 436, 440 et 442 ). D'habitude, près de l'entrée, à gauche et à droite, il y avait une petite pièce fermée par des murs à piliers et sol généralement pavé de pierres (Chambon, I984: 158-160). La maison $n^{\circ}$ I76 avait deux pièces avec des murets à piliers sur le côté gauche, qui s'ouvraient sur l'espace central du bâtiment, comme cela a été attesté à la « Maison $A$ »(Chambon, I984: 162).

\section{Datation absolue}

Lors des campagnes 2018 et 20I9, plusieurs échantillons de charbon de bois ont été collectés pour datation absolue de la « Maison A » de Tell el-Far'a. Parmi ceux-ci, six échantillons (pris des pièces $n^{\circ} \mathrm{I}$ 


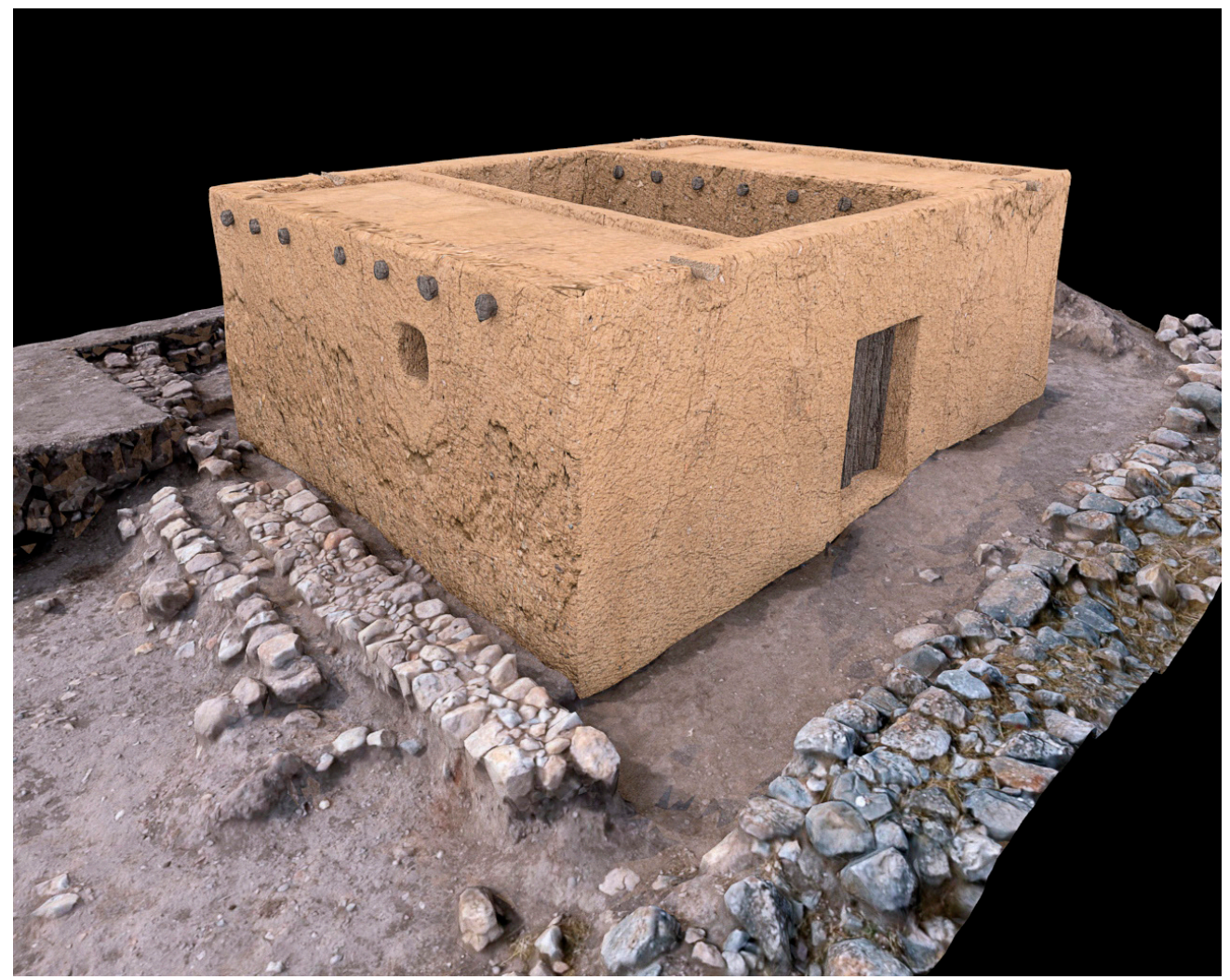

Figure 9. Hypothèse de reconstruction en 3D de la « Maison A ». Vue extérieure (J.G. Gómez, TEF)

Figure 9. Hypothetical reconstruction in 3D of "House A". External view (J.G. Gómez, TEF)

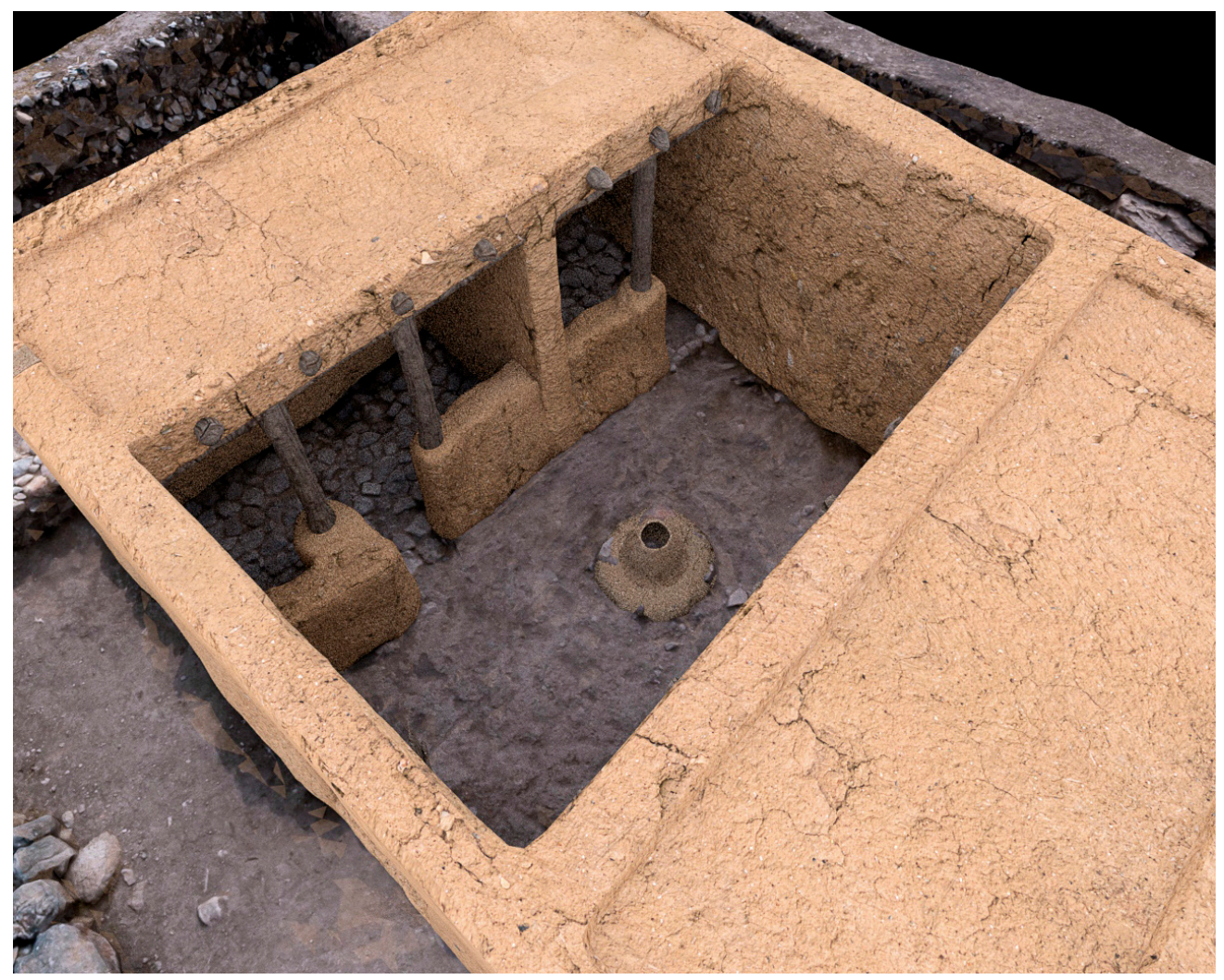

Figure 10. Hypothèse de reconstruction en 3D de la « Maison A ». Vue de l'espace central (J.G. Gómez, TEF)

Figure 10. Hypothetical reconstruction in 3D of "House A". View of the central area (J.G. Gómez, TEF) 


\begin{tabular}{|c|c|c|c|c|c|}
\hline $\begin{array}{l}\text { Échantillon et } \\
\text { campagne }\end{array}$ & Contexte & Code Lab & Âge ${ }^{14} \mathrm{C}$ & Calib 10 BCE & Calib $2 \sigma \mathrm{BCE}$ \\
\hline$M 6 / 2018$ & Pièce $n^{\circ}$ 2. Sol UC.114 & $18 \mathrm{C} / 1267$ & $2840 \pm 30$ & $990 \pm 54$ & $1013 \pm 96$ \\
\hline Tranche d'âge & & & & $1043-936$ & $1108-917$ \\
\hline M5/2018 & Pièce $n^{\circ}$ 2. Sol UC.114 & $18 \mathrm{C} / 1266$ & $2780 \pm 30$ & $938 \pm 42$ & $924 \pm 80$ \\
\hline Tranche d'âge & & & & $980-896$ & $1004-844$ \\
\hline$M 4 / 2018$ & Pièce n 2. Sol UC.114 & $18 \mathrm{C} / 1265$ & $2910 \pm 30$ & $1117 \pm 72$ & $1110 \pm 99$ \\
\hline Tranche d'âge & & & & $1188-1045$ & $1209-1011$ \\
\hline M9/2019 & $\begin{array}{l}\text { Pièce } n^{\circ} 1 . \text { Près de la porte du } \\
\text { mur UC.120 }\end{array}$ & $19 \mathrm{C} / 1201$ & $3420 \pm 30$ & $1710 \pm 44$ & $1754 \pm 118$ \\
\hline Tranche d'âge & & & & $1754-1666$ & $1871-1636$ \\
\hline $\mathrm{M} 12 / 2019$ & Pièce n 1. Tannour UC.123 & $19 \mathrm{C} / 1202$ & $2790 \pm 30$ & $948 \pm 44$ & $934 \pm 78$ \\
\hline Tranche d'âge & & & & $992-904$ & $1011-856$ \\
\hline M8/2019 & $\begin{array}{l}\text { Pièce } n^{\circ} 1 . \text { Près de la marmite } \\
\text { 19.A1.103.16 }\end{array}$ & $19 \mathrm{C} / 1203$ & $2850 \pm 30$ & $996 \pm 55$ & $1019 \pm 92$ \\
\hline Tranche d'âge & & & & $1051-941$ & $1111-927$ \\
\hline
\end{tabular}

Tableau 1. Résultats de la datation AMS de charbons de bois obtenus à Tell el-Fa'ra lors des campagnes 2018 et 2019

Table 1. AMS dating results through carbonized wood at Tell el-Far‘a (2018 and 2019 seasons)

et $\mathrm{n}^{\mathrm{O}}{ }_{2}$ ) ont été datés par la méthode de spectrométrie de masse par accélérateur (AMS) au laboratoire ICA (International Chemical Analysis Inc.) à Sunrise (Floride). Les résultats des dates $\mathrm{C}^{\mathrm{I} 4}$ avec les calibrations individuelles correspondantes et les intervalles de confiance I $\sigma$ et $2 \sigma$ sont présentés ci-dessous dans le tableau I. Les calibrations ont été obtenues en utilisant la courbe de Reimer et alii (20I3) et Oxcal (Bronk Ramsey et Lee, 2013: 720-730).

\section{Discussion}

L'étude d'ensemble de la stratigraphie, de l'architecture, de la céramique et de la datation ${ }^{\mathrm{I}}{ }^{\mathrm{C}} \mathrm{C}$ de la "Maison A » de Tell el-Far'a ouvre de nouvelles perspectives sur la chronologie absolue de l'âge du Fer II dans les montagnes centrales de Palestine. La comparaison des dates absolues obtenues à Tell el-Far'a (campagnes 2018-2019) avec d'autres datations faites précédemment (2006-2019), montre l'absence de coïncidence pour la phase VIIb du site (selon la dénomination des archéologues français).

La proposition chronologique du tableau 2 faite par les archéologues israéliens se fonde exclusivement sur des comparaisons typologiques des céramiques trouvées dans les fouilles de l'École biblique à Tell el-Far'a. Ainsi, Z. Herzog et L. Singer-Avitz
(2006: I74-I76 et I85-186) considèrent que la céramique de la période VIIb de Tell el-Far'a est typologiquement comparable à celle de Megiddo VA-IVB, qu'ils datent du «Late Iron Age IIA » (début du IX ${ }^{\mathrm{e}}$ siècle à $840 / 830$ av. J.-C.). Ces céramiques correspondent à ce qu'ils appellent le " Jezreel Cluster ». De même, I. Finkelstein (20I2: 334 et 338), à partir de l'étude de la céramique de Tell el-Fara VIIb, a conclu qu'elle appartient à l'horizon Megiddo VA-IVB et a proposé une datation dans le « Late Iron IIA » (ca. 870-deuxième moitié du Ix ${ }^{\mathrm{e}}$ siècle av. J.-C.). A. Kleiman (20I8: 95 et 99) a réétudié la céramique de la phase VIIb du site de Far'a et a assuré qu'elle est contemporaine aux niveaux Q-5 de Megiddo et V de Tel Rehov (datés par radiocarbone à la fin du $\mathrm{x}^{\mathrm{e}}$ siècle av. J.-C., ou au début du $\mathrm{IX}^{\mathrm{e}}$ siècle av. J.-C.). Récemment, Finkelstein et Kleiman (2019: 29I) ont proposé dater Tell el-Far'a VIIb dans le " Middle Iron IIA » (ca. 900 av. J.-C., à l'époque du roi Basha).

Entre 2006 et 20I2, les archéologues israeliens ont souligné que les céramiques de la période VIIa de Tell el-Far'a présentent des analogies avec celles trouvées à Megiddo VB et ont suggéré de dater cette période ou bien du " Early Iron IIA », deuxième moitié du $\mathrm{x}^{\mathrm{e}}$ siècle av. J.-C. (Herzog et Singer-Avitz, 2006: I85-I86), ou bien du « Late Iron I-Early Iron IIA », de la deuxième moitié du $\mathrm{x}^{\mathrm{e}}$ siècle av. J.-C. au début du IX ${ }^{\mathrm{e}}$ siècle av. J.-C. (Finkelstein, 20I2: 334). 


\begin{tabular}{lllllll}
\hline $\begin{array}{l}\text { Tell } \\
\text { el-Far'a }\end{array}$ & $\begin{array}{l}\text { Herzog, } \\
\text { Singer-Avitz } \\
\text { (2006) }\end{array}$ & Finkelstein (2012) & Kleiman (2018) & $\begin{array}{l}\text { Finkelstein, } \\
\text { Kleiman (2019) }\end{array}$ & $\begin{array}{l}\text { Plus récente } \\
\text { (2018-2019) }\end{array}$ & $\begin{array}{l}\text { Moyenne } \\
\text { pondérée } \\
\text { (2018-2019) }\end{array}$ \\
\hline VIllb & $\begin{array}{l}900-840 / 830 \\
\text { av. J.-C. }\end{array}$ & $\begin{array}{l}\text { 870-deuxième } \\
\text { moitié du IXe siècle } \\
\text { av. J.-C. }\end{array}$ & $\begin{array}{l}\text { Fin Xe siècle- } \\
\text { début IXe siècle } \\
\text { av. J.-C. }\end{array}$ & ca. 900 av. J.-C. & $987-939$ av. J.-C. & 1004-959 av. J.-C. \\
& & & & \\
\hline
\end{tabular}

Tableau 2. Intervalles chronologiques pour la phase VIIb de Tell el-Far'a, proposés par différents auteurs et comparés aux données obtenues (1б) par AMS (campagnes 2018 et 2019)

Table 2. Chronological gaps concerning period VIlb at Tell el-Far'a, indicated by several scholars and compared with the obtained data (10) through AMS (2018 and 2019 seasons)

Selon I. Finkelstein (20I2: 338 et 346), Tell el-Far'a VIIa (une modeste colonie non fortifiée d'environ un hectare) correspondrait chronologiquement à l'époque de la ville de Tirza, capitale du royaume du nord.

De son côté, A. Chambon (1984: 12), tout en reconnaissant que la stratigraphie de Tell el-Far'a doit être réexaminée, a proposé de dater la période $\mathrm{VIIb}$ du site au $\mathrm{X}^{\mathrm{e}}$ siècle av. J.-C., mais il a suggéré que ses débuts pourraient être au $\mathrm{XI}^{\mathrm{e}}$ siècle av. J.-C. Cette datation était fondée à nouveau sur des comparaisons typologiques de la poterie avec d'autres sites de la région du Levant (Megiddo, Taanak, Hazor, etc.).

Les travaux menés à bien entre 2017 et 2019 par le nouveau projet international à Tell el-Far'a ont ouvert de nouvelles perspectives sur la question de la datation absolue de l'âge du Fer dans la région. Pour le moment, il ne s'agit que de cinq dates par radiocarbone (AMS) obtenues pour cette période, mais ce sont les premières que nous avons de ce site clé pour avancer sur la question complexe de la chronologie absolue en Palestine au début du Ir $^{\text {er }}$ millénaire av. J.-C. Bien que les données ne doivent pas être considérées comme définitives, elles indiquent une date du $\mathrm{X}^{\mathrm{e}}$ siècle av. J.-C. pour la période VIIb («Maison A ) du site. L'échantillon M9/2org a donné une datation plus ancienne, correspondant au Bronze moyen II (période V de Tell el-Far'a). Il doit s'agir d'un cas du « old wood effect ", c'est-à-dire l'échantillon provienne d'un morceau de bois ancien réutilisé (Finkelstein, 2019: 28).

\section{Conclusion}

La stratigraphie, l'architecture et le mobilier céramique de la « Maison A » de Tell el-Far'a VIIb ne laissent aucun doute quant à son attribution à l'âge du Fer IIA. Une autre question plus complexe est la datation absolue de cette période, que certains chercheurs ont daté du IX ${ }^{\mathrm{e}}$ siècle av. J.-C. Cependant, les datations au radiocarbone réalisées en 2018 et 2019 (les premières faites du site) par la nouvelle équipe montrent une datation au $\mathrm{x}^{\mathrm{e}}$ siècle av. J.-C. (cf. tableaux I et 2). Si cette datation absolue est ratifiée, et précisée davantage encore par l'étude de nouveaux échantillons, la phase VIIb, et non la phase VIIa de Tell el-Far'a (comme cela a été proposé), serait celle qui pourrait correspondre chronologiquement à la ville de Tirza. Un autre problème encore plus difficile à clarifier est celui de savoir si Tell el-Far'a est, en effet, l'ancienne cité (mentionnée jusqu'à dix-sept fois dans l'Ancien Testament) où a résidé Jéroboam ${ }^{\text {er. }}$.

\section{Remerciements}

Le projet désire remercier la Fondation Palarq, le CHAM-Centre de Sciences Humaines (UNL), l'équipe de recherche CulXeo (UDC), le Consulat général d'Espagne à Jérusalem et l'AECID (Ministère des Affaires étrangères, Union européenne et coopération d'Espagne) pour le soutien apporté. De même, les auteurs souhaitaient exprimer sa gratitude au Ministère de Tourisme et des Antiquités de Palestine, à la municipalité du wadi el-Faráa, au Centre sportif et de la jeunesse du Camp de Far'a et à l'Institut biblique et archéologique espagnol (Casa de Santiago) de Jérusalem pour leur soutien et leur collaboration pendant les campagnes réalisées. 


\section{Bibliographie}

Abdulfattah, K. et De Vries, B. eds. (2005): Wadi el-Far'a project report: an environment assessment of the Wadi el-Far'a watershed. BRIC. Bergen.

Abel, F.M. (1938): Géographie de la Palestine. Paris.

Ahituv, S. (1984): Canaanite toponyms in Ancient Egyptian Documents. Brill. Leiden.

Albright, W.F. (193I): "The site of Tirzah and the Topography of Western Manasseh”. Journal of the Palestine Oriental Society, II: 24I-25I.

Alt, A. (1927): "Ophra". Palästinajabrbuch, 23: 36-38. Alt, A. (1932): "Die Reise”. Palästinajabrbuch, 28: 40-44. Amiet, P., Briend, J., Courtois, L. et Dumortier, J.B. (1996): Tell el-Far'ah. Histoire, glyptique et céramologie. Éditions Universitaires / Vandenhoeck $\&$ Ruprecht. Fribourg.

Ben-Tor, A., Zarzecki-Peleg, A. (2015): "Iron Age IIA-B: Northern valleys and Upper Galilee”. In S. Gitin (ed.): The ancient pottery of Israel and its neighbors from the Iron Age through the Hellenistic Period. Israel Exploration Society. Jerusalem: 135-188.

Bronk Ramsey, C. et Lee, S. (2013): "Recent and planned developments of the program oxCal". Radiocarbon, 55(2-3): 720-730.

Bryce, T. (20I2): "Farah, Tell el". In T. Bryce: The Routledge Handbook of the Peoples and Places of Ancient Western Asia. Routledge. London-New York: 240-24I.

Burke, A. (2008): Walled up to Heaven. The Evolution of Middle Bronze Age Fortification Strategies in the Levant. Winona Lake. Harvard.

Chambon, A. (1984): Tell el-Far'ah I. L'âge du Fer. Éditions Recherche dur les Civilisations. Paris.

Charloux, G. (2005-2006): "Production des céramiques et évolution sociale en Palestine de la fin $\mathrm{du} 4^{\mathrm{e}}$ au $3^{\mathrm{e}}$ millénaire av. J.-C.". Techniques et Culture, 46-47: 273-283.

Conder, C.R. et Kitchener, H.H. (1882): The survey of western Palestine. Vol. II: Samaria. London.

Dababsa, I., de Wall, K. et Rjoob, A. (2005): "Sustainable development of the Wadi al-Far'a: archaeology sector report". In K. Abdulfattah et B. De Vries (eds.): Wadi el-Far'a project report: an environment assessment of the Wadi el-Far'a watershed. BRIC. Bergen: 77-99.
De Vaux, R. (I95Ia): "La troisième campagne de fouilles à Tell el-Far'ah, près Naplouse". Revue Biblique, 58: 393-430.

De Vaux, R. (1951b): "La troisième campagne de fouilles à Tell el-Far'ah, près Naplouse. Rapport préliminaire (suite)". Revue Biblique, 58: 566-59o.

De Vaux, R. (1952): "La quatrième campagne de fouilles à Tell el-Far'ah, près Naplouse. Rapport préliminaire". Revue Biblique, 59: 551-583.

De Vaux, R. (1955): "Les fouilles de Tell el-Far'ah, près Naplouse. Cinquième campagne. Rapport préliminaire". Revue Biblique, 62: 54I-589.

De Vaux, R. (1956): “The Excavations at Tell el-Far'ah and the Site of Ancient Tirzah". Palestine Exploration Quarterly, 88: I25-I40.

De Vaux, R. (1957): "Les fouilles de Tell el-Far'ah, près Naplouse. Sixième campagne. Rapport préliminaire". Revue Biblique, 64: 552-580.

De Vaux, R. (196r): "Les fouilles de Tell el-Far'ah. Rapport préliminaire sur les $7^{\mathrm{e}}, 8^{\mathrm{e}}, 9^{\mathrm{e}}$ campagnes, 1958-1960". Revue Biblique, 68: 557-592.

De Vaux, R. (I962): "Les fouilles de Tell el-Far'ah. Rapport préliminaire sur les $7^{\mathrm{e}}, 8^{\mathrm{e}}, 9^{\mathrm{e}}$ campagnes, 1958-1960 (suite)". Revue Biblique, 69: 212-253.

De Vaux, R. (1967): “Tirzah”. In D.W. Thomas (ed.): Archaeology and Old Testament Study. Clarendon Press. Oxford: $37 \mathrm{I}^{-}-383$.

De Vaux, R. (1976): “El-Far'a, tell, North”. In M. AviYonah (ed.): Encyclopedia of Archaeological Excavations in the Holy Land. Vol. 2. Israel Exploration Society and Massada Press. Jerusalem: 395-404.

De Vaux, R., Miroschedji, P. de, et Chambon, A. (1993): "Far'ah, Tell el- (North)".In E. Stern (ed.): The New Encyclopedia of Archaeological Excavations in the Holy Land. Vol. 2. The Israel Exploration Society. Jerusalem: 433-440.

De Vaux, R. et Steve, A.M. (I947a): "La première campagne de fouilles à Tell el-Far'ah, près Naplouse. Rapport préliminaire". Revue Biblique, 54: 394-433.

De Vaux, R. et Steve, A.M. (1947b): "La première campagne de fouilles à Tell el-Far'ah, près Naplouse. Rapport préliminaire (suite)". Revue Biblique, 54: 573-589.

De Vaux, R. et Steve, A.M. (1948): "La seconde campagne de fouilles à Tell el-Far'ah, près Naplouse. Rapport préliminaire”. Revue Biblique, 55: 544-580. 
De Vaux, R. et Steve, A.M. (1949): "La deuxième campagne de fouilles à Tell el-Far'ah, près Naplouse. Rapport préliminaire (suite)". Revue Biblique, 56: IO2-I38.

Dever, W.G. (200I): What did the biblical writers know and when did they know it? Wm. B. Eerdmans Publishing Co. Cambridge.

Fernández-Tresguerres, J.A. (1987): "La industria de piedra tallada de Tell el-Far'ah, norte”. In V. Collado et V. Vilar (eds.): II Simposio Bíblico Español. Monte de Piedad y Caja de Ahorros de Córdoba. Valencia: 15-29.

Finkelstein, I. (20I2): "Tell el-Far'ah (Tirzah) and the early days of the Northern Kingdom". Revue Biblique, II9: 331-346.

Finkelstein, I. (2013): Le royaume biblique oublié. Odile Jacob. Paris.

Finkelstein, I. (20I9): "L'histoire d'Israël ancien entre archéologie et texte biblique. État des lieux". In I. Finkelstien et Th. Römer (eds.): Aux origines de la Torah. Nouvelles rencontres, nouvelles perspectives. Bayard. Paris: $\mathbf{5 5} 55$.

Finkelstein, I. et Kleiman, A. (2019): "The archaeology of days of Baasha?”. Revue Biblique, I26: 277-296.

Glueck, N. (1951): Explorations in Eastern Palestine, IV. Part I: Text. American Schools of Oriental Research. New Haven.

Guérin, V. (1874): Description géographique, historique et archéologique de la Palestine, seconde partie, Samarie. Paris.

Herzog, Z. (1997): Archaeology of the city. Urban planning in ancient Israel and its social implications. Institute of Archaeology/Tel Aviv University. Tel Aviv.

Herzog,Z. et Singer-Avitz, L. (2006): "Sub-dividing the Iron Age IIA in Northern Israel: a suggested solution to the chronological debate". Tel Aviv, 33: 163-195.

Huot, J.L. (1967): "Typologie et chronologie relative de la céramique du Bronze ancien à Tell el-Fâ'rah”. Revue Biblique, 74: 517-554.

Ilan, Z. (1973): The Jordan valley and the desert of Samaria. Tel Aviv (en hébreu).

Jasmin, M. (2013): “Tell el-Far'ah (N)". In D.M. Master (ed.): The Oxford Encyclopedia of the Bible and Archaeology. Vol. 2. Oxford University Press. Oxford: 393-400.
Jasmin, M. (2020): "Les royaumes d'Israël et de Juda ( $\mathrm{x}^{\mathrm{e}}-\mathrm{vi} \mathrm{I}^{\mathrm{e}}$ siècle av. J.-C.)". In M. Sauvage (dir.): Atlas historique du Proche-Orient ancien. Les Belles Lettres. Paris: I20.

Kappus, S. (1966): “Oberflachenuntersuchungen im Mittlren Wadi Far'ah”. Zeitschrift des Deutschen Palästina-Vereins, 82: 74-82.

Kellermann, D., Mittmann, S., Schmitt, G. et Zwickel, W. (1992): "Palestine. Iron Age settlement (ca. I200-550 BC)”. TAVO B IV-6. Tübingen.

Kelley, J.L. (2014): The 'black' juglets in Iron Age II Palestine: a collation of finds and an analysis of typology, chronology and distribution. Jerusalem University College. Jerusalem.

Kenyon, K. M. (1979): Archaeology in the Holy Land. Routledge. London-New York.

Kitchen, K. A. (1973): The third intermediate period in Egypt. Aris \& Phillips. Warminster.

Kleiman, A. (2018): "Comments on the archaeology and history of Tell el-Far'ah north (biblical Tirzah) in the Iron IIA". Semitica, 60: 85-IO4.

Knierim, R. (1969): "Oberflachenuntersuchungen im Wadi Far'a”. Zeitschrift des Deutschen Palästina-Vereins, 65: 51-62.

Kochavi, M. ed. (1972): Judea, Samaria ant the Golan. Archaeological Survey 1967-I968.Jerusalem (en hébreu).

Laato, A. (2015): Guide to biblical chronology. Sheffield Phoenix Press. Sheffield.

Lipinski, E. (2018): A bistory of the Kingdom of Israel. Peeters. Leuven.

Mallet,J.(1973): Tell el-Fa'rah (Jordanie). L'installation du Moyen Bronze antérieure au rempart. Gabalda. Paris.

Mallet, J. (1987): Tell el-Fâr'ab II. Le Bronze Moyen, 2 vols. Éditions Recherche Sur Les Civilisations. Paris.

Marcus, A.D. (2000): The view from Nebo. How archaeology is rewriting the Bible and reshaping the Middle East. Little, Brown and Company. Boston-New York-London.

Medeghini, L., Sala, M., De Vito, C. et Mignardi, S. (2019): "A forgotten centre of ceramic production in Southern Levant: Preliminary analytical study of the Early Bronze Age pottery from Tell el-Far'ah North (West Bank)". Ceramics International, 45: II457-II467. 
Miroschedji, P. de (1976): Contribution à l'étude de l'urbanisation en Palestine à l'âge du Bronze Ancien. Thèse de doctorat. Paris.

Miroschedji, P. de (200I): "Les 'maquettes architecturales' palestiniennes”. In B. Muller (ed.): «Maquettes architecturales» de l'Antiquité. Diffusion de Boccard. Paris: 43-85.

Miroschedji, P. de (2013): "Les villes de Palestine de l'âge du Bronze Ancien à l'âge du Fer dans leur contexte proche-oriental". Cabiers des Thèmes Transversaux Arscan, XI: 185-198.

Montero Fenollós, J.L., Caramelo, F., Yasin, J. et Dias, S. (2019): "Nuevo proyecto arqueológico en Tell el-Fara, Palestina. Informe preliminar de la primera campaña (2017)". Aula Orientalis, 37/r: IO5-II9.

Montero Fenollós, J.L., Caramelo, F., Yasin, J., Dias, S. et Sanjurjo, J. (2020a): "Investigaciones arqueológicas en Tell el-Far'a, Palestina. Informe preliminar de la segunda campaña (2018)". Aula Orientalis, 38/2: 335-349.

Montero Fenollós, J.L., Caramelo, F., Yasin, J., Sanjurjo, J. et Bejarano, I. (2020b): "Investigaciones arqueológicas en Tell el-Far'a (Palestina). Informe preliminar de la tercera campaña (20I9): excavación, prospección, toponimia y restauración”. Res Antiquitatis, 2: 104-I3I.

Nigro, L., Fiacavento, Ch. et Yasine, J. (2015): "Archaeology from A to Z: Abu Zarad, an ancient town in the heartland of Palestine". Vicino Oriente, I9: I39-I83.

Pardo, J., Montero, J.L., Caramelo, F., Yasin, J et Gómez,J.G. (2021): Tell el-Far'a. Arqueología en Palestina. 20I7-20Ig. Sociedade Luso-Galega de Estudos Mesopotámicos. Ferrol.
Picard, C. (1958): "Sur une figurine de bronze argenté trouvée à Tell el-Far'ah, près de Naplouse (Palestine)". Revue Archéologique, r: 91-93.

Pola, Th., Kröger, H., Rasink, B., Reinhard, J., al-Balawnah, M. et Abu Abila, M. (20r6): "A Preliminary Report of the Tulul adh-Dhahab (wadi az-Zarqa). Survey and Excavation Seasons 200520II". Annual of the Department of Antiquities of Jordan, 57: 8I-96.

Robinson, E. (184I): Biblical researches in Palestine, Mount Sinai and Arabia Petraea. Vol. 3. Crocker and Brewster. Boston.

Sapir, Y. (199I): “Tell el-Far'ah-Tirzah?”. Samaria and Benjamin, II: 197-206 (en hébreu).

Sharkas, O., Abu Hammad, A., Vanden Berg, B.A., Blankespoor, B. et Naslund, S. (2005): "Natural landscapes”. In K. Abdulfattah et B. De Vries (eds.): Wadi el-Far' a project report: an environment assessment of the Wadi el-Far'a watershed. BRIC. Bergen: I4-49.

Sussman, V. (2007): Oil-Lamps in the Holy Land: Saucer Lamps. From the beginning to Hellenistic period. Collection of the Israel Antiquities Authority. British Archaeological Reports International Series, 1598. Archaeopress. Oxford.

Reimer, P.J. et alii (2013): "IntCaliz and Marinerz radiocarbon age calibration curves $0-50,000$ years cal BP”. Radiocarbon, 55: 1869-1887.

Tappy, R.E. (2015): “Iron Age IIA-B: Samaria”. In S. Gitin (ed.): The ancient pottery of Israel and its neighbors from the Iron Age through the Hellenistic Period. Israel Exploration Society. Jerusalem: 189-2II.

Wittke, A.M., Olshausen, E. et Szydlak, R. eds. (20I2): Historischer Atlas der antiken Welt. Metzler. Stuttgart. Zertal, A. (2008): The Manasseh Hill Country Survey. Vol. 2: The Eastern Valleys and Fringes of the Desert. Brill. Leiden-Boston. 